\title{
Edge Chipping Minimisation Strategy for Milling of Monocrystalline Silicon: A Molecular Dynamics Study
}

\author{
Zi Jie Choong1, Dehong Huo ${ }^{1 *}$, Patrick Degenaar², Anthony O'Neill²
}

${ }^{1}$ Mechanical Engineering, School of Engineering, Newcastle University, Newcastle Upon Tyne, NE1 7RU, UK ${ }^{2}$ Electrical and Electronic Engineering, School of Engineering, Newcastle University, Newcastle Upon Tyne, NE1 $7 \mathrm{RU}, \mathrm{UK}$

*Corresponding author: Tel: +44 191208 6230, email: dehong.huo@ newcastle.ac.uk

\begin{abstract}
Direct patterning of functional microstructures on monocrystalline silicon by mechanical micro-milling has drawn intense interests as an alternative to the conventional lithographybased technique in microelectronics fabrication. Despite the micro milling offers advantages such as versatility and low operating cost, machining induced defects such as edge chipping occur on the surface edges of a finished product and may affect its functionality. To address such challenge, a novel hybrid technique that combines mechanical machining and the deposition of a layered sacrificial structure on the silicon surface was proposed to minimise the machining induced edge chipping. In this paper, the cutting mechanism of silicon under the proposed hybrid technique has been studied by molecular dynamics simulation. Fundamental mechanisms such as material deformation, chip formation and stress behaviour are analysed. Reduction of stress intensity and machining forces has been observed when silicon was machined under the proposed hybrid conditions. The effect is believed to be contributed by thermal softening, resulted from the high cutting temperature and interfacial stress between the copper and silicon layers. In addition, machining silicon by the proposed hybrid technique also showed desirable properties which include low subsurface damages, large material removal rate and better surface finishing $\left(R_{a}<0.1 \mathrm{~nm}\right)$. The results further enriched the machining theory and justified the feasibility for the proposal of such a hybrid technique.
\end{abstract}

Keywords: Nanometric machining, monocrystalline silicon, edge chipping, layered sacrificial structure, material deformation 


\section{Introduction}

Fabrication of products from monocrystalline silicon, a hard and brittle material, at micro-scale level can be challenging when good machining quality and high accuracy are required [1,2]. Currently, subtractive techniques such as DRIE and wet etching are used $[3,4]$. Although they achieve patterned subtraction by a lithography process, they are only economical for large-scale manufacture and are limited to planar structures. In contrast, mechanical micro-milling offers simple setup with high accuracy and 3D machining capability. Recent advancements had shown the success of mechanical micro-milling to shape monocrystalline silicon with exceptional surface quality by enabling ductile mode cutting conditions [5-10]. Such conditions usually involve stringent control of the machining parameters to generate a highly compressive hydrostatic pressure in the cutting zone to suppress crack propagation [11-12].

Mechanical micro-milling is a maskless technique, which allows direct machining of functional structures on silicon's surface. It is also an economical alternative and helps to bridge the gap of micro/mesoscale components fabrication for prototyping or small batch production. Despite its versatility and low operating cost, excessive generation of defects such as edge chipping may affect the product's functionality. Furthermore, the machining parameters that enabled the ductile mode cutting conditions in the previous literature did not provide mechanical machining with an advantageous over the conventional micro-electro-mechanical systems (MEMs) fabrication methods or non-traditional hybrid techniques in terms of the material removal rate. This was due to the use of extremely small feed rate and axial depth of cut ranging between the nano and sub-micron scales to enable ductile mode cutting.

Edge chipping is generated along the surface edges of a machined profile. It is common when silicon is mechanically machined at tens to hundreds of microns in depth. Concerns over the presence of edge chipping usually associate with the disruption to the functionality of the final machined product, since subsequent cutting passes cannot remove them. There have been ongoing efforts to improve the machinability of brittle materials by mechanical machining. Some of which involves the hybrid of different techniques which combines mechanical micromilling with the assistance of other methods such as vibration, thermal (laser), chemical and ion implantation [13-17].

Due to the ubiquitous usage of monocrystalline silicon in the semiconductor and MEMs industrial sectors, the existence of surface electronics and its complex layout requirements may hinder the productive employment of existing hybrid techniques to its fabrication. Several 
limitations include the metallurgical transformation of surface properties and challenges in achieving complex machining trajectories when shaping of advanced geometries [18-20].

To address the describe issues, an alternate hybrid manufacturing technique is proposed. The proposed hybrid technique uses mechanical micro-milling as the primary process and combines a suitable deposition method to construct a ductile sacrificial layer structure above the brittle silicon substrate. The sacrificial layer structure helps to create an energy buffer between the cutting tool and the brittle silicon substrate and intends to absorb any high impact energy generated during contact. The sacrificial layer also allows any edge chipping to form on its body rather than on the brittle silicon substrate, thus reducing the brittleness of silicon and improving its elasticity.

Moreover, the use of mechanical machining does not limit the choice of material during the material removal process. In particular, its incorporation as the primary shaping technique allows the selectivity of the material options to be independent of the removal method. This eliminates the need for any chemical or gas etching by DRIE, hence, reducing the number of photolithography steps, time and cost. The proposed hybrid technique also aims to preserve the flexibility and versatility of mechanical micro-milling while preventing unnecessary changes to the material surface properties of the finished product upon machining.

Considering its novelty and the challenge to investigate the mechanism of edge chipping mitigation by experimental approach at the nanometric level, this study aims to provide these insights and its feasibility using molecular dynamics (MD) simulation. The organisation for the rest of this paper is as follows: Firstly, Section 2 discusses the simulation methods such as the techniques used in the construction of the simulation model and its associated simulation parameters. The cutting mechanism during silicon machining by the proposed hybrid technique are then discussed in Section 3. Subsequently, post machining analysis such as the subsurface damages and nanometric surface morphology are analysed by the qualitative and quantitative measurements on the simulated silicon workpiece. Finally, the conclusions of this paper are drawn in Section 5.

\section{Simulation Method}

In this study, MD simulation was conducted to study the mechanism of employing a ductile sacrificial layer structure during nanometric cutting of silicon. The ductile sacrificial layer structure to be used in the proposed hybrid technique is preferably soft with good machinability, easy to deposit and remove. Some examples may include non-ferrous metals, which are better 
in corrosion resistance and can be readily electroplated, and polymers, which can be easily spun or sputtered onto the materials to be machined.

As MD simulation is primarily developed around open source programs, two essential factors for the successful employment of the simulation models were considered. The first factor relates to the ease of constructing the respective ductile sacrificial structure suitable for the MD simulation. It directly dictates the computational effort required for the intended investigation. Although polymers like Polymethylmethacrylate (PMMA) and SU-8 negative epoxy resin can be readily deposited onto silicon, construction of material models for MD will require additional pre-processing software and material databases.

The second factor, which is also the most crucial to an MD simulation, is the availability of a suitable potential energy function to describe the interaction between the silicon and the intended ductile material. Considering the described factors and that the principal objective for this study is to understand the edge chipping mitigation mechanism by employing a ductile sacrificial layer structure rather than the influence of the sacrificial material type, copper was chosen to represent the ductile sacrificial layer structure for simplicity. Figure 6-1(a) shows the MD model established for an orthogonal machining process with a constant uncut chip thickness. It consists of an amorphous copper layer, a (001) monocrystalline silicon substrate and a monocrystalline diamond tool.

\section{(a)}

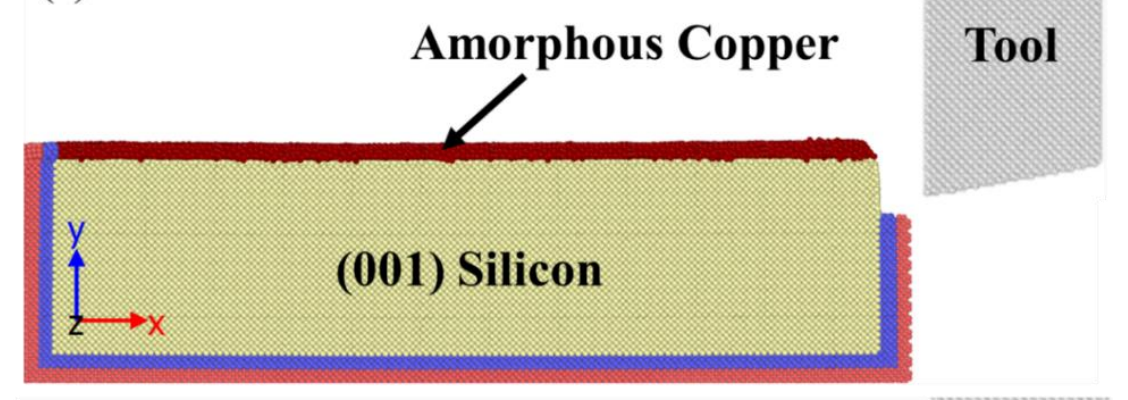

(b) Newtonian Layer

Thermostat Layer

Boundary Layer

Tool

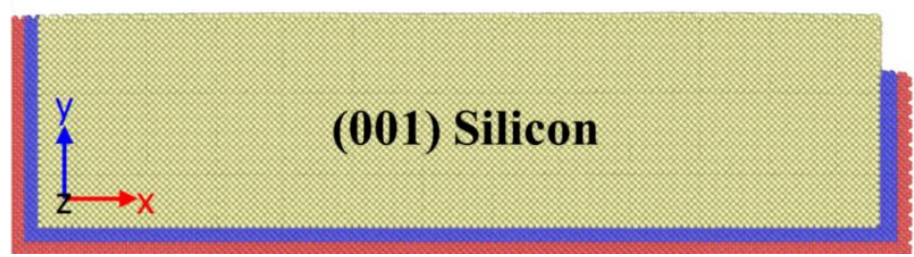

Figure 0-1: (a) Ductile copper sacrificial layer and (b) uncoated monocrystalline silicon MD simulation models. 
To construct the amorphous copper layer, a "melt and quench" method was used for the transformation from its bulk crystalline form. The amorphous copper structure was built using the NPT ensemble, and the monocrystalline copper atoms were initialised with a face-centered cubic (FCC) crystal lattice structure. After being melted by a temperature of $2000 \mathrm{~K}$, the processed copper atoms were rapidly quenched to $300 \mathrm{~K}$ at a cooling rate of $10^{12} \mathrm{~K} / \mathrm{s}$.

Radial distribution function (RDF) analysis was used to verify the amorphous copper structure after its construction by the "melt and quench" technique as described above. Figure 6-2 shows the calculated RDF of the monocrystalline and amorphous copper structure after the rapid quenching process. As increasing temperature changes the copper lattice structure, characteristics of the amorphous copper were confirmed by the absence of a long-range order of the monocrystalline copper structure and a second peak splitting feature at $r=4.5$ [345-346].
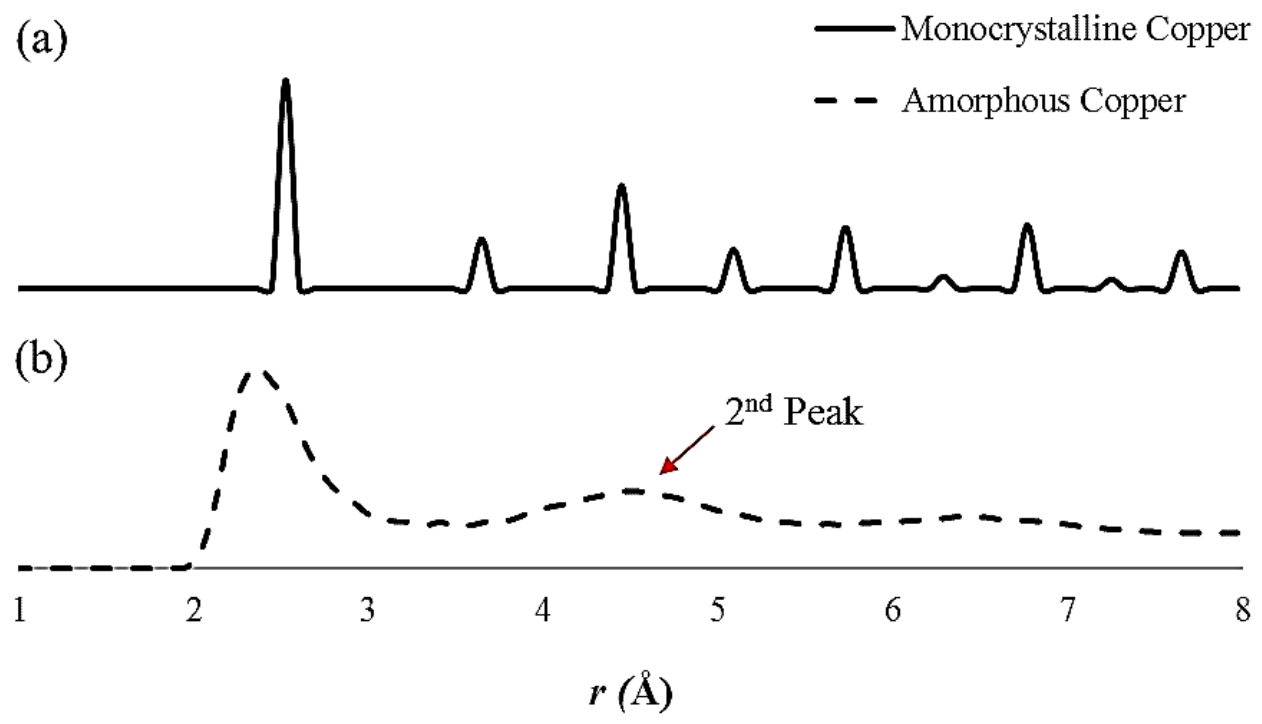

\section{Figure 0-2: RDF of the ductile copper sacrificial layer (a) monocrystalline and (b) amorphous structure.}

Regarding the simulated workpiece construction, the amorphous copper was integrated onto the surface of a (001) monocrystalline silicon substrate. The cutting region containing the amorphous copper structure and monocrystalline silicon will be addressed as the amorphouscrystalline composite region for discussion purposes. Periodic boundary conditions (PBC) were applied to all three dimensions ( $\mathrm{X}, \mathrm{Y}$ and $\mathrm{Z}$ ) to avoid any surface effects. The workpiece contains three different layers, namely the Newtonian, thermostat and boundary. The boundary layer was fixed around the workpiece to prevent any unnecessary movement and maintain its symmetry during cutting. 
The thermostat layer lies between the boundary and Newtonian layers and helps to dissipate the cutting heat. Since the nanometric cutting model is extremely small and not capable of dispersing the cutting heat itself, the thermostat layer is periodically re-scaled during simulation. The thickness of both the boundary and the thermostat layers were constructed at approximately 0.6 $\mathrm{nm}$. Due to the periodical rescaling of the thermostat layer, the workpiece temperature was maintained at $300 \mathrm{~K}$ throughout the whole simulation process. Consequently, atoms located within the Newtonian layer region will be subjected to the equation of motion by Newton's second law and assume a micro-canonical (NVE) ensemble during the cutting process [240].

The cutting tool was modelled with a $0^{\circ}$ nominal rake angle to resemble a monocrystalline diamond micro-end-mill used in the experiment. It was modelled as a rigid body, and the effect of tool wear was not considered. Cutting tools with an atomically sharp and rounded cutting edges were also constructed to compare its influence on the cutting performances. Furthermore, an uncoated monocrystalline silicon model which depicts normal machining conditions was also created for comparison purposes as shown in Figure 6-1(b).

For the selection of a suitable potential energy function, its importance and the corresponding parameters for describing the atomic interactions will be crucial to predict the properties of the material accurately. Currently, there are numerous multi-body empirical potentials constructed to represent both the covalent (silicon) and metallic (copper) systems. A widely employed embedded-atom method (EAM) potential proposed by Daw and Baskes [347] was chosen to represent the copper atoms in this study. The EAM potential gives a realistic description of the behaviour and properties of a metallic bonded material. The algorithm which the EAM potential uses to describe the total energy of atom $i, E_{i}$, located within the metallic system is given by Equation 6-1.

$$
E_{i}=F_{\alpha_{e a m}}\left(\sum_{j \neq i} \rho_{e a m} \beta_{e a m}\left(r_{i j}\right)\right)+\frac{1}{2}\left(\sum_{j \neq i} \varphi_{e a m} \alpha_{e a m} \beta_{e a m}\left(r_{i j}\right)\right)
$$

Where:

- $F_{\alpha_{e a m}}$ is the embedding energy which is a function of the atomic electron density, $\rho_{\text {eam }}$.

- $\varphi_{\text {eam }}$, represents a pair of the potential interaction.

- $\quad \alpha_{\text {eam }}$ and $\beta_{\text {eam }}$ are the element types of atoms $I$ and $J$ respectively.

In this paper, interactions between the amorphous copper atoms were represented by the EAM potential, and its corresponding parameters were explicitly parametrised by Adams et al. [348]. Subsequently, the SW and ABOP potentials were employed to represent the interactions 
between the silicon workpiece, carbon (diamond tool) and their interatomic bonding respectively [ref]. For the interaction between the silicon and the copper atoms, two potential energy functions, namely the modified embedded-atom method (MEAM) potential (an extension of the EAM potential) and the charge-optimized many-body (COMB) were initially considered [349-350]. These potentials consider the interaction between the covalently bonded systems with its metallic counterpart and can be readily used for the silicon-copper structure.

Despite the availability of the potential energy functions, it is worth noting that the employment of either MEAM or COMB potentials to the nanometric cutting simulation will significantly increase the computational effort when compared to traditional potentials such as EAM and Tersoff. An indicative example adopted from the benchmarked results of different potential energy functions by previous literature and LAMMPS suggest that the COMB potential will require at least 25 and 14 times more computation efforts and cost when compared to the EAM and traditional Tersoff potential energy functions respectively [252].

Fortunately, Zhang et al. [351] recently parametrised a Tersoff potential energy function to represent the interaction between the silicon and copper atoms. As such, the modified Tersoff possible energy function by Zhang et al. was adopted. Before simulating in dynamics conditions, all simulated models were subjected to energy and thermal equilibrium to achieve steady state in both energy and thermal conditions. Simulations were conducted by the large-scale atomic/molecular massively parallel simulator (LAMMPS) [252], while the results were visualised by OVITO [253]. Detailed simulation conditions are listed in Table 1. Model 1-6 are the simulation models under normal or hybrid conditions using different cutting edge radius, $r_{e}$, and cutting directions (see Table 1)

Table 0-1: MD simulation conditions used in this study.

\begin{tabular}{|c|c|c|}
\hline \multirow{4}{*}{$\begin{array}{l}\text { Geometrical } \\
\text { Parameters }\end{array}$} & Si-Cu Model & $\begin{array}{l}\text { Silicon: } 33.786(x) \times 8.803(y) \times 5.431(z) \mathrm{nm} \\
\text { Copper: } 33.786(x) \times 0.543(y) \times 5.431(z) \mathrm{nm}\end{array}$ \\
\hline & $\begin{array}{l}\text { Monocrystalline } \mathrm{Si} \\
\text { Model }\end{array}$ & $33.786(x) \times 9.346(y) \times 5.431(z) \mathrm{nm}$ \\
\hline & Diamond Tool & $6.931(x) \times 9.103(y) \times 5.431(z) \mathrm{nm}$ \\
\hline & Diamond Tool Geometry & $\begin{array}{c}\text { Rake Angle: } 0^{\circ} \\
\text { Clearance Angle: } 10^{\circ} \\
\text { Cutting Edge Radius, } r_{e}: 0.08 \text { and } 1.5 \mathrm{~nm}\end{array}$ \\
\hline \multirow{3}{*}{$\begin{array}{l}\text { Machining } \\
\text { Parameters }\end{array}$} & Uncut Chip Thickness & $1.5 \mathrm{~nm}$ \\
\hline & $\begin{array}{l}\text { Machining Feed } \\
\text { Directions }\end{array}$ & (001) [100] and (001) [110] \\
\hline & $\begin{array}{c}\text { Total Tool Travel } \\
\text { Distance }\end{array}$ & $25 \mathrm{~nm}$ (including $1.3 \mathrm{~nm}$ free travel) \\
\hline
\end{tabular}




\begin{tabular}{|c|c|c|}
\hline & Cutting Velocity & $100 \mathrm{~m} / \mathrm{s}$ \\
\hline \multirow{2}{*}{ MD Parameters } & MD Timestep & $1 \mathrm{fs}$ \\
\hline & Total Timesteps & 250,000 steps \\
\hline \multirow{5}{*}{$\begin{array}{l}\text { Potential } \\
\text { Energy } \\
\text { Functions }\end{array}$} & $\mathrm{Cu}-\mathrm{Cu}$ & Embedded-atom Method (EAM) \\
\hline & $\mathrm{Si}-\mathrm{Si}$ & Modified Stillinger-Weber (SW) \\
\hline & $\mathrm{Si}-\mathrm{Cu}$ & Modified Tersoff \\
\hline & $\mathrm{Cu}-\mathrm{C}[352]$ & Morse $^{1} \rightarrow D_{o}: 0.087 \mathrm{eV} ; \alpha: 5.14 \AA^{-1} ; r_{0}: 2.05 \AA$ \\
\hline & $\mathrm{Si}-\mathrm{C}$ and $\mathrm{C}-\mathrm{C}$ & Analytic Bond-Order Potentials (ABOP) \\
\hline \multirow{6}{*}{$\begin{array}{c}\text { Simulation } \\
\text { Model } \\
\text { Configurations }\end{array}$} & Model 1 & Normal condition, $r_{e}=0.08 \mathrm{~nm},[100]$ \\
\hline & Model 2 & Normal condition, $r_{e}=1.5 \mathrm{~nm},[100]$ \\
\hline & Model 3 & Proposed hybrid condition, $r_{e}=0.08 \mathrm{~nm},[100]$ \\
\hline & Model 4 & Proposed hybrid condition, $r_{e}=0.08 \mathrm{~nm},[110]$ \\
\hline & Model 5 & Proposed hybrid condition, $r_{e}=1.5 \mathrm{~nm},[100]$ \\
\hline & Model 6 & Proposed hybrid condition, $r_{e}=1.5 \mathrm{~nm},[110]$ \\
\hline
\end{tabular}

\section{Nanometric Cutting Mechanism of Silicon under the Proposed Hybrid Condition}

\subsection{Machining Stress and Temperature}

The ability of a brittle material to deform plastically during mechanical material removal can significantly affect the cutting performance and final machining quality of the finished product. To better understand the material deformation process, the equivalent local atomic strain, hydrostatic stress and Tresca maximum shear stress were proposed to assess the yielding criterion of the monocrystalline silicon during nanometric cutting process under both the normal and proposed hybrid conditions. Although machining was conducted along the [100] and [110] directions to study the anisotropic behaviour of monocrystalline silicon, similar machining trends were observed in the [100] and [110] directions during machining. As such, only the effect of machining along the machining feed direction of [100] direction was discussed for brevity.

Figure 6-5 shows the equivalent local atomic shear strain of the selected simulation models. When machining monocrystalline silicon under normal conditions, a thinner PDSZ and higher concentration of shear strain gather ahead of the tool cutting edge when extremely small cutting edge radius was used. The effect can be observed by comparing between Models 1 and 2 (Figures 6-5(a) and 6-5(b)) and agrees with the shear plane angle calculations in Figure 4-27. However, with the introduction of a ductile sacrificial layer, atomic shearing within the tool

\footnotetext{
${ }^{1} D_{o}$ is the dissociation energy of the bond, $\alpha$ is the scaling length, and $r_{0}$ is the equilibrium interatomic distance.

${ }^{2}$ Permutations of the machining parameters and tool geometries are encoded for concision in the discussion. The encoding follows the order of machining environment, tool cutting edge radius and machining feed direction.
} 
cutting edge region weakens. The effect was demonstrated by Models 3 and 5 as shown in Figures 6-5(c) and 6-5(d).

To further understand the material deformation under the influence of the proposed hybrid machining condition, both the hydrostatic and maximum shear stresses were analysed, and its distribution within the silicon workpiece during the nanometric cutting process at the tool travel distance of $20 \mathrm{~nm}$ are shown in Figures 6-6 and 6-7 respectively. During the machining of silicon under the normal conditions, atoms situated within the cutting zone experience high hydrostatic stress peaking at about $-13 \mathrm{GPa}$ and exhibit high mobility phase similar to the fluid flow. The effect is demonstrated by the blue region (negative stress value) as shown in Figures 6-6(a) and 6-7(b).
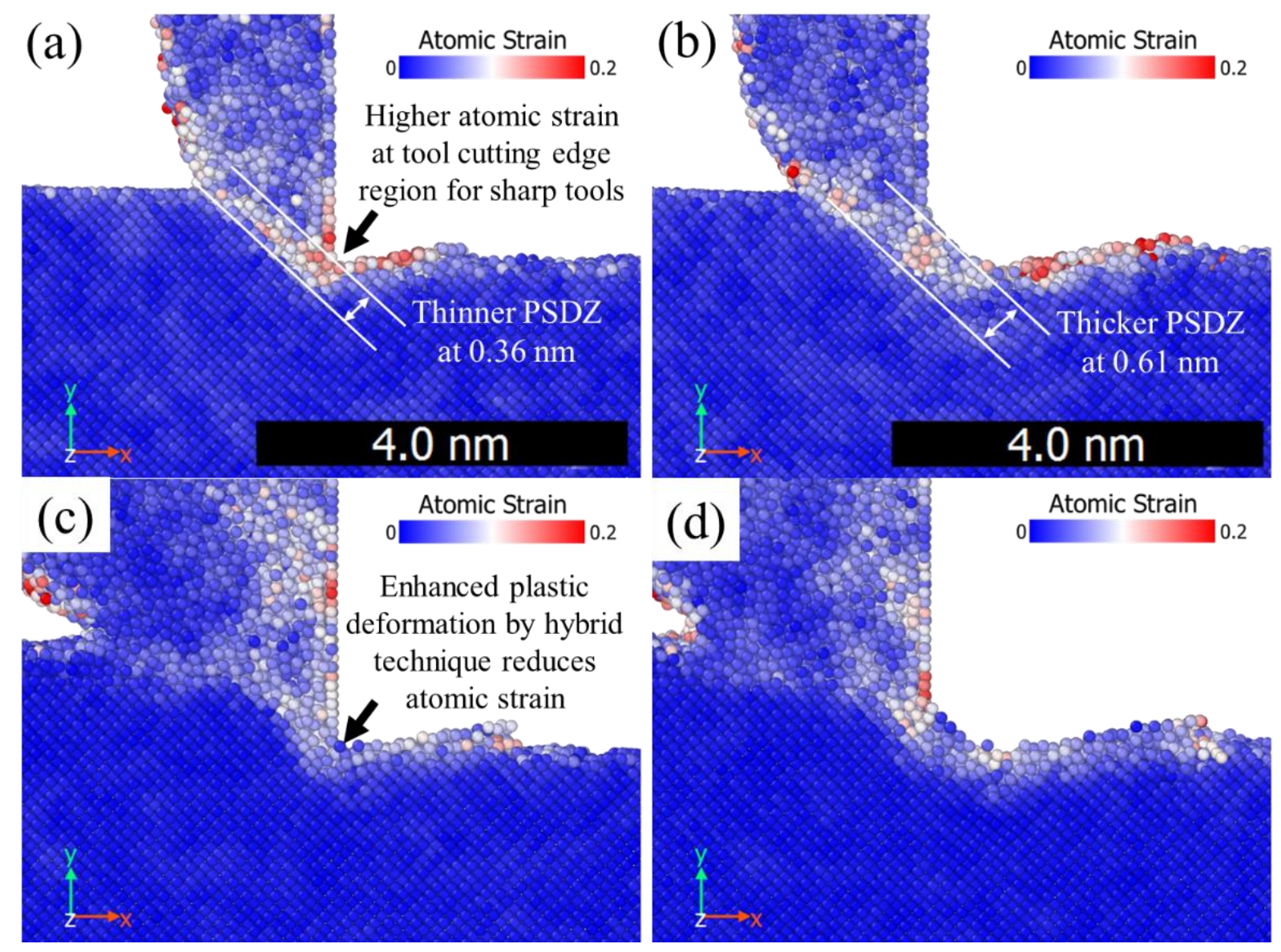

Figure 0-3: Atomic strain distribution at tool advancement distance of $20 \mathrm{~nm}$ on (a) Model 1, (b) Model 2, (c) Model 3 and (d) Model 5.

Due to the highly compressive action of the tool's cutting edge, the cubic diamond lattice structure type with fourfold coordination is exhaustively destroyed, thus leading to a phase transformation. Plastic deformation occurs readily within this region and is a crucial process to achieve ductile mode cutting in the machining of brittle silicon. As the tool cutting edge radius depicted in Model 2 is larger than Model 1, higher negative effective rake angle will be achieved 
by the cutting tool in Model 2 under similar cutting conditions. Such an effect can cause higher induction of compressive action within the cutting zone, which leads to a more extensive distribution area of the hydrostatic stress in Model 2 (Figure 6-6(b)). Materials within the silicon workpiece that are situated outside the compressive region maintain its hardness and stiffness.

As shown in Figure 6-7(b), a structural phase boundary occurs between the amorphous fluid like silicon structure and its crystalline bulk counterpart within the cutting zone. As the plastic removal process continues during the tool travel, atoms that are within a fluid phase structure will flow up towards the rake face of the cutting tool to form chips. Due to the increased velocity gradient contributed by the plastic flow during chip formation, the phase boundary experiences violent shear strain and friction, thus promoting elastic deformation and concentration of shear stress within the crystalline bulk region.

Tensile stress is also an essential factor in the formation and propagation of a crack [358]. Maximum principal stress was employed for the assessment due to its capability to identify tension. Results depicting the maximum principal stress at the tool travel distance of $20 \mathrm{~nm}$ for silicon nanometric cutting under the normal and proposed hybrid conditions are shown in Figure 6-8. 

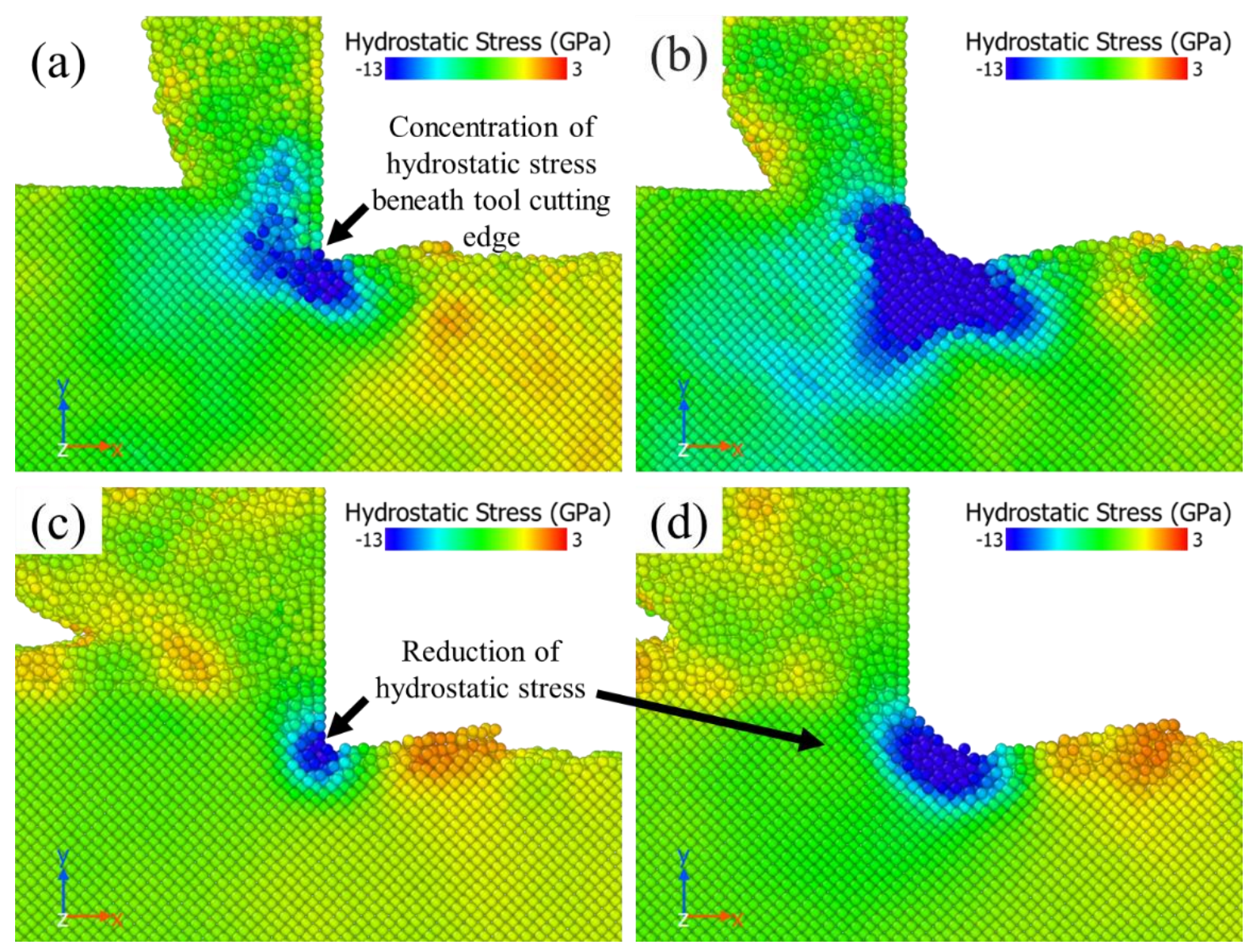

Figure 0-4: Hydrostatic stress distribution at tool advancement distance of $20 \mathrm{~nm}$ on (a) Model 1, (b) Model 2, (c) Model 3 and (d) Model 5. 

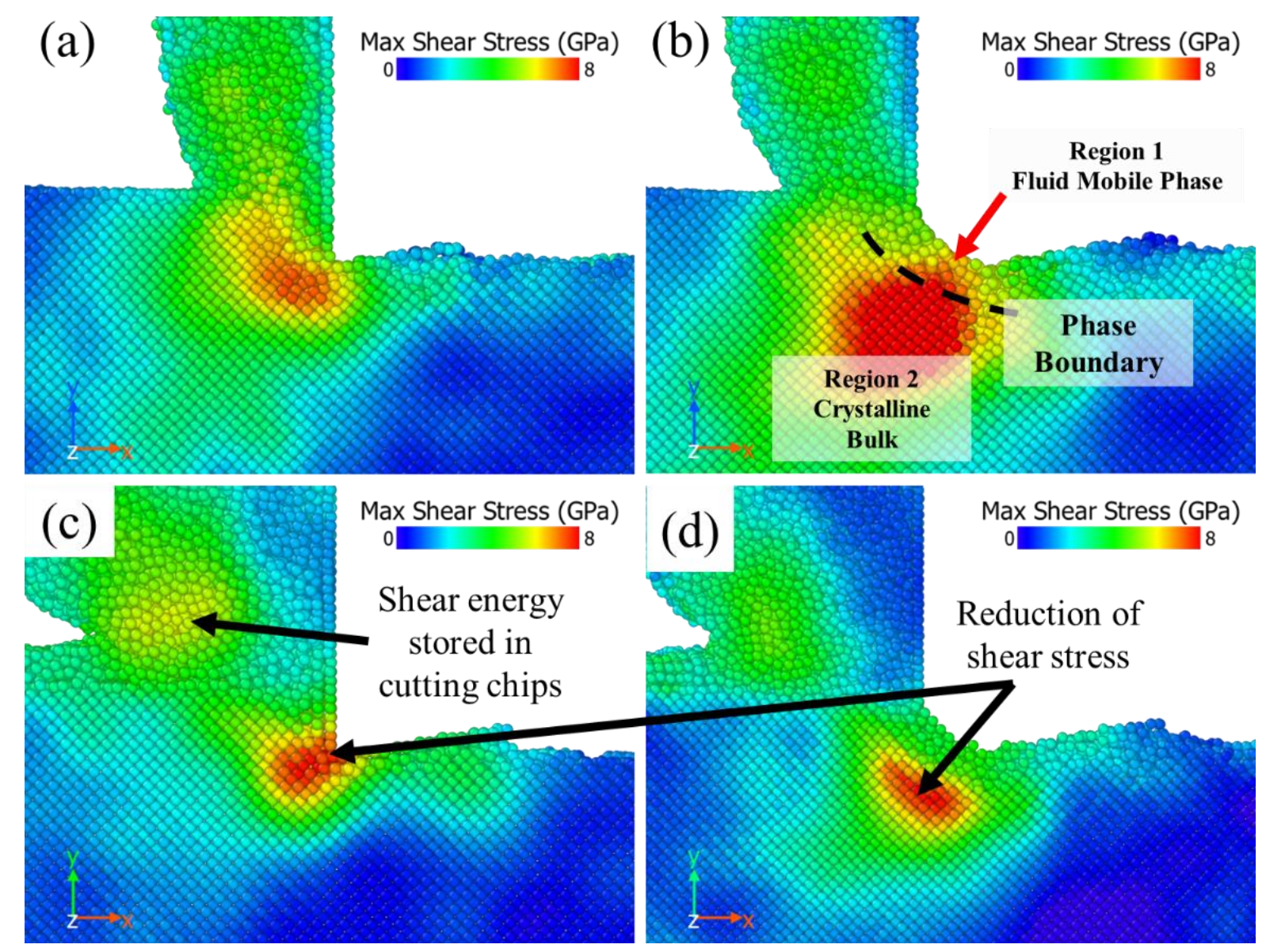

Figure 0-5: Tresca maximum shear stress distribution at tool advancement distance of $20 \mathrm{~nm}$ on (a) Model 1, (b) Model 2, (c) Model 3 and (d) Model 5.

In the case of normal machining condition, distribution of the maximum principal stresses in Figures 6-8(a) and 6-8(b) show that plastic flow denoted by the negative value was exhibited at the PSDZ due to compression by the tool cutting edge. As such, no fracture will occur within this vicinity as no tensile forces were present. However, a high level of tensile stress is observed to occur especially underneath the tool flank region and the area ahead of the tool cutting edge. Since tensile stress is responsible for the initiation of fracture in brittle materials, micro-cracks can originate from these areas during micromachining, thus resulting in edge chipping.

On the contrary, the dominance of ductile characteristics contributed by the amorphous copper structure in the amorphous-crystalline composite region influenced the crystalline bulk silicon to behave like the amorphous silicon. The hardness of silicon within the amorphous-crystalline interface region was also believed to be reduced using a ductile sacrificial layer structure. Therefore, the effect enhances the plastic deformation of silicon during machining and leads to a significant reduction in the hydrostatic (Figures 6-6(c) and 6-6(d)) and shear stresses (Figures 6-7(c) and 6-7(d)). Moreover, tensile stress was also reduced (Figures 6-8(c) and 6-8(d)) when 
monocrystalline silicon was machined by the proposed hybrid technique, regardless of the machining feed directions and tool geometries.

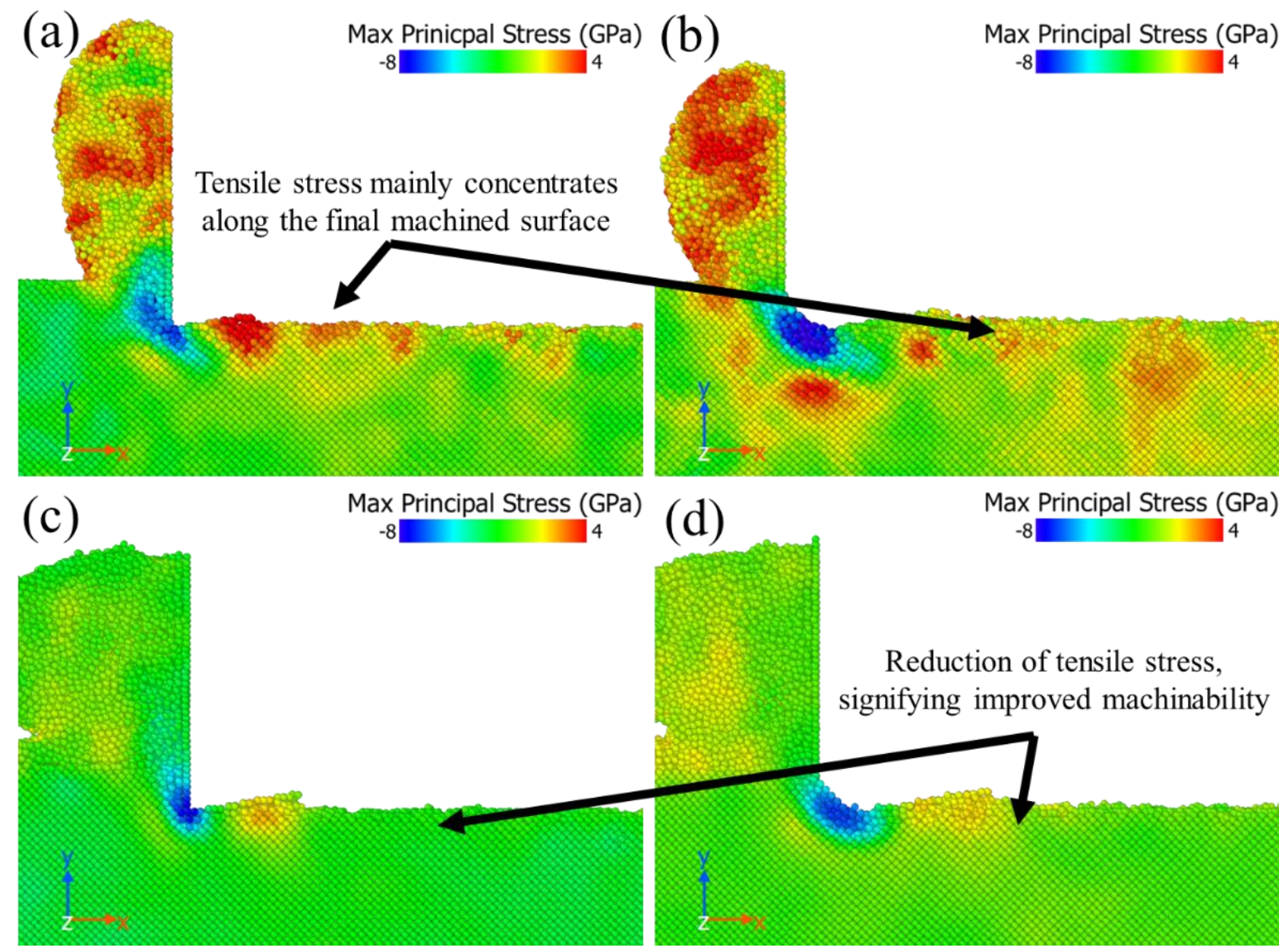

\section{Figure 0-6: Maximum principal stress distribution at tool advancement distance of 20} nm on (a) Model 1, (b) Model 2, (c) Model 3 and (d) Model 5.

As the ductile sacrificial layer was believed to reduce the hardness of silicon within the amorphous-crystalline composite cutting region, atoms within the cutting zone will more likely be swept up to form the chips rather than being compressed into the subsurface. Hence the residual tensile stresses were stored in the chips, which in turn reduces the energy encapsulation within the workpiece. Furthermore, the amorphous-crystalline structure deforms better than the ideal crystalline bulk silicon. Therefore, the velocity gradient and the atomic strain in the PSDZ as shown in Figures 6-5(c) and 6-5(d) were also reduced.

Apart from the material deformability, thermal softening of the silicon workpiece by the heat generated during machining also contributes to the mechanism of plasticity enhancement. The vast differences in thermal conductivity between the amorphous copper sacrificial layer and the monocrystalline silicon workpiece cause high interfacial stress to occur [359]. Therefore, higher temperature was observed when silicon nanometric cutting was conducted under the proposed 
hybrid conditions. The thermal effect was demonstrated by selected examples in Models 1 and 3 as shown in Figures 6-9(a) and 6-9(b) respectively.
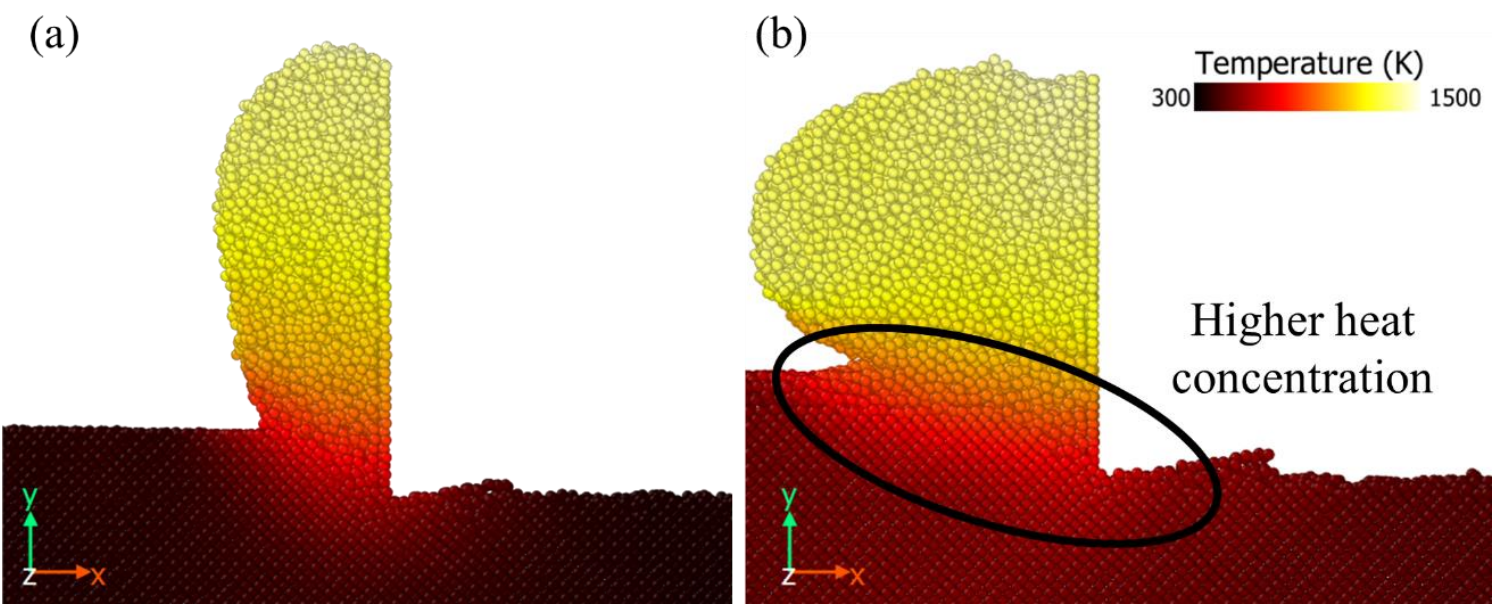

Figure 0-7: Machining temperature distribution contour at tool advancement distance of $20 \mathrm{~nm}$ on (a) Model 1 and (b) Model 3.

\subsection{Machining Forces}

Analysis of the machining forces can provide further insights into the influences of the tool cutting edge radius, the use of a ductile sacrificial layer structure and machining feed directions during silicon nanometric cutting. Figures 6-10, 6-11 and 6-12 show the thrust, $F_{t}$, and the cutting, $F_{c}$, forces of selected simulated models. Figure 6-13 also shows the average resultant machining forces computed across all simulation models at a tool travel distance of $20 \mathrm{~nm}$ for more explicit depiction. In general, a distinctive trend can be observed when monocrystalline silicon was machined under the proposed hybrid condition.

As shown in Figures 6-10 and 6-11, the oscillation amplitude of the force signals for Models 3 and 5 (hybrid conditions) was observed to be smaller than those generated in Models 1 and 2 (normal conditions) respectively. Due to the reduction of hydrostatic stresses as shown in the previous section, the thrust force, $F_{t}$, generated in Models 3 and 5 were also observed to be lower than its respective counterparts in Models 1 and 2, when machined under normal conditions. Such behaviour correlates well with the stress analysis as discussed previously. Moreover, comparing the resultant forces for Models 3 and 5 with their respective counterparts in Models 1 and 2 respectively, it was evident that a significant reduction of $35 \%$ and $23 \%$ can be achieved when monocrystalline silicon was machined by cutting tool with extremely small and rounded cutting edge respectively under the proposed hybrid condition.

Another interesting phenomenon was observed on the force behaviour in Figures 6-12(a) and 6-12(b). Although higher machining forces were generated when machining along the [110] 
direction of a (001) surface silicon, the anisotropic effect on the machining forces was greater reduced when a ductile sacrificial layer was employed during machining. The observation was beneficial to demonstrate the technique's capability of minimising the peculiarity of silicon's anisotropic influence at an early stage. The resultant forces showed such an effect in Figure 613, where an insignificant increment of $3 \%$ was observed when comparing the machining forces between [110] in Model 4 and [100] in Model 3.
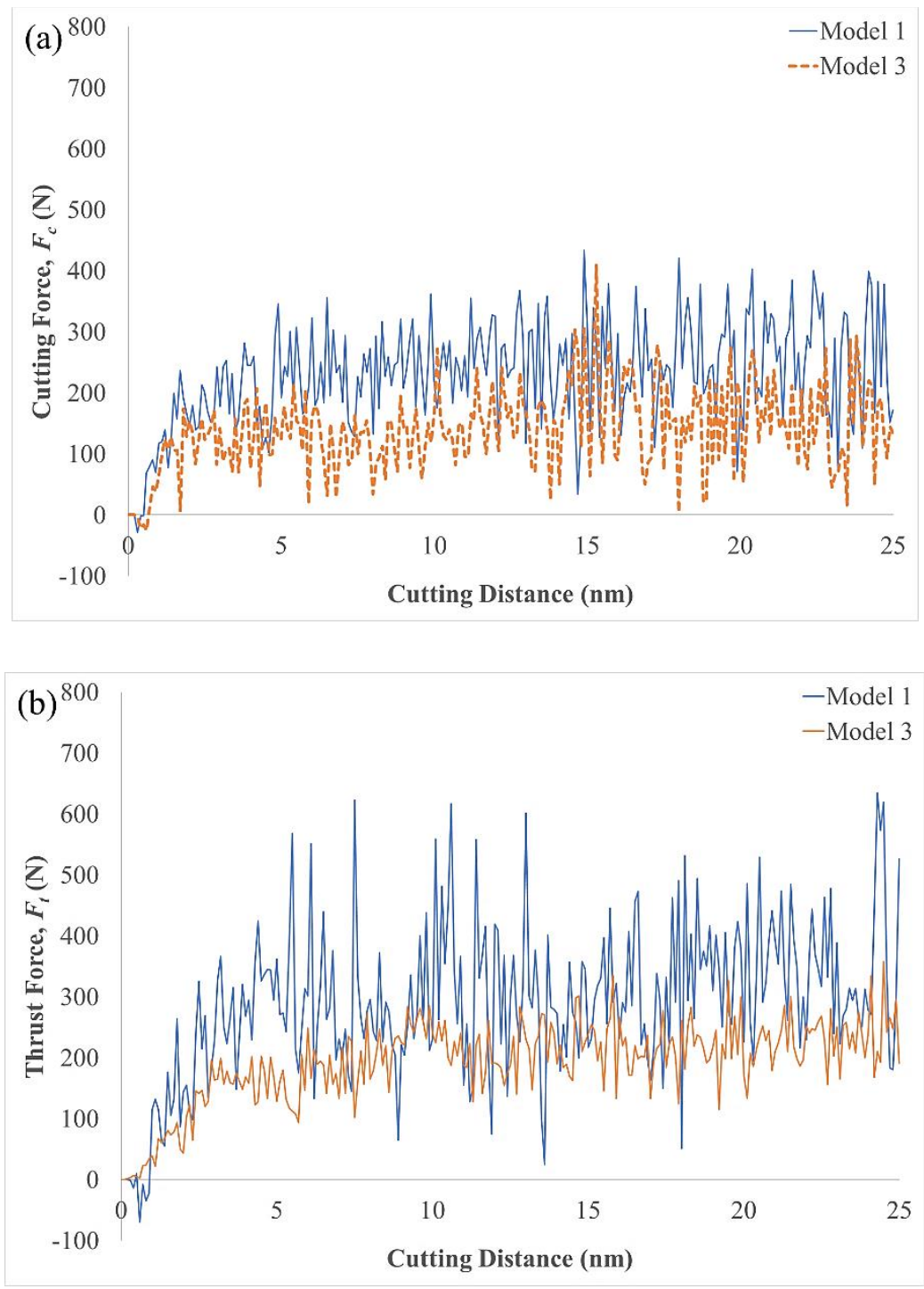

Figure 0-8: Influence of the machining technique by the atomically sharp cutting tool on the (a) cutting force, $F_{c}$, and (b) thrust force, $F_{t}$, demonstrated by comparing between Models 1 and 3. 

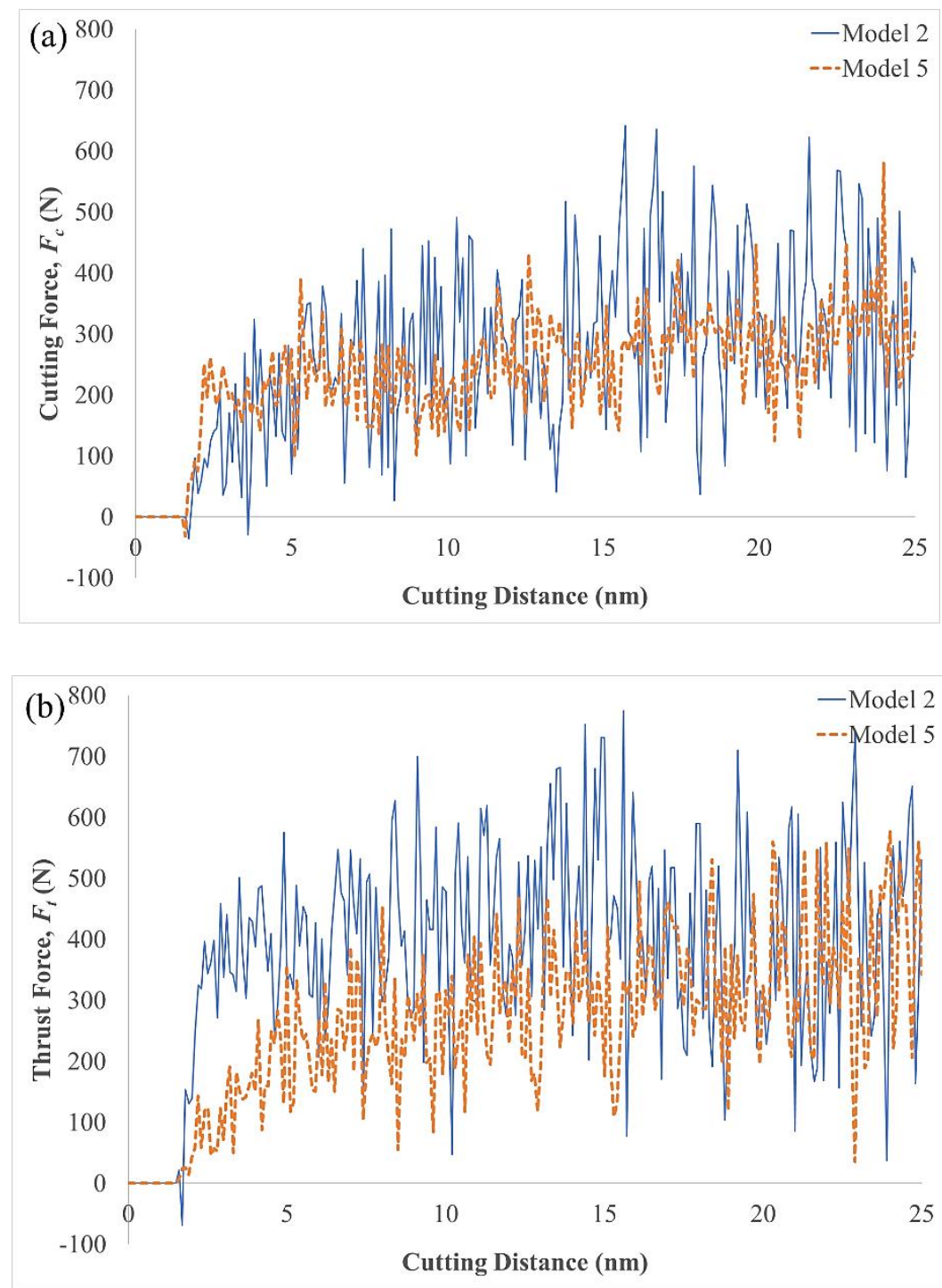

Figure 0-9: Influence of the machining technique by rounded cutting edge tool on the (a) cutting force, $F_{c}$, and (b) thrust force, $F_{t}$, demonstrated by comparing between Models 2 and 5.

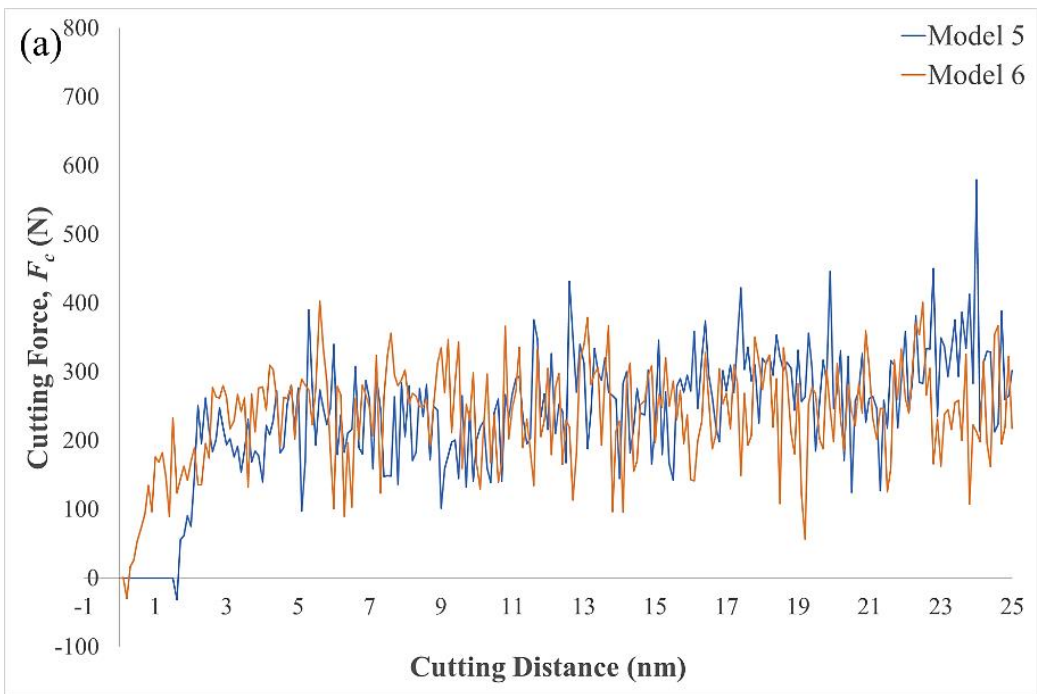




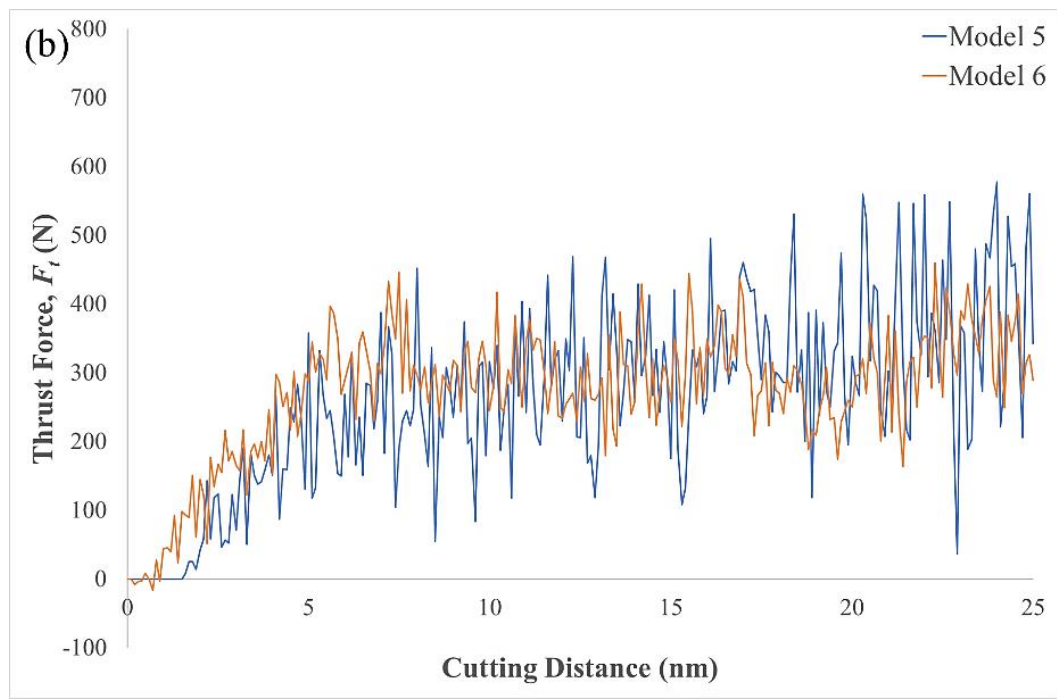

Figure 0-10: Influence of the machining feed direction (defect) on the (a) cutting force, $F_{c}$, and (b) thrust force, $F_{t}$, demonstrated by the comparison between Models 5 and 6.

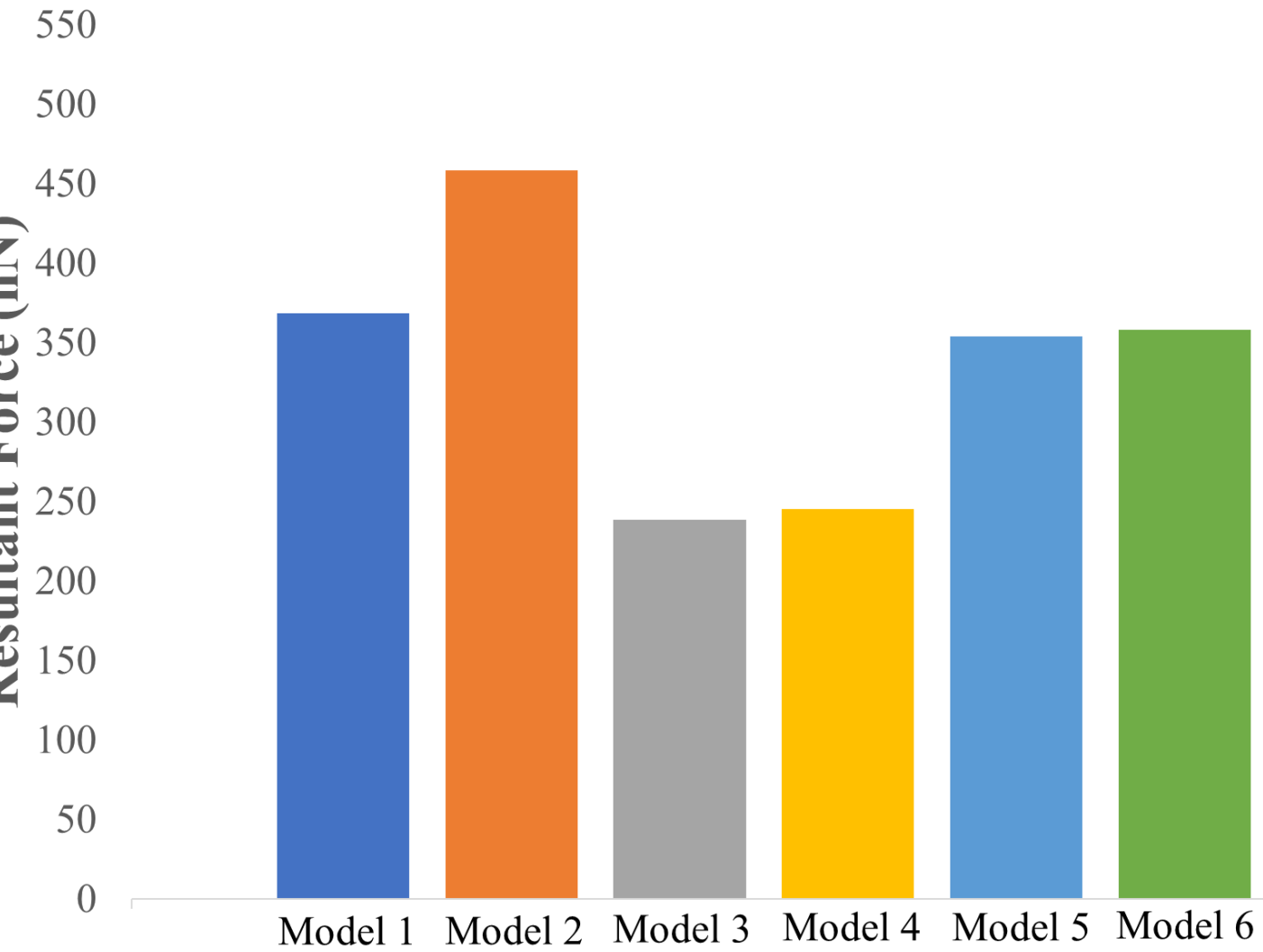

Figure 0-11: Average resultant force computed for all simulation models. 


\section{Post-Machining Analysis}

Apart from the analysis on the machining stress, temperature and forces, assessment on several post-machined performances such as the chips, subsurface deformation, subsurface damage and surface roughness can also help to understand the cutting behaviour of the proposed hybrid technique. These performances were measured at the end of tool travel at $25 \mathrm{~nm}$.

\subsection{Chip Morphologies}

A selected analysis will be discussed in this section for brevity, due to similar trends observed in other simulated models. Figure 6-14 shows the selected chip morphologies of Models 2 and 5. As seen, thicker chip structure was generated in Model 5 when compared to Model 2. In theory, larger chip thickness generally correlates with a lower shear plane angle and higher machining forces. The effect also signifies greater cutting resistance and poor machining quality. However, such a theory cannot be applied in this context to assess the material deformation and removal performance for the amorphous-crystalline composite region, as described in the proposed hybrid technique. The reason is that the chips as shown in Model 5 (Figure 6-14(b)) contain both the copper and silicon elements, and the theory contradicts with the reported machining stress and forces observed from the previous sections.

Considering such effect, counting of the number of silicon chip atoms was proposed instead of characterising the material removal performance for nanometric cutting of silicon under both normal and proposed hybrid condition. Figure 6-15 shows the selected number of silicon chip atoms measured from simulation Models 2 and 5. From the observation, it was evident that more silicon atoms were present in the chip zone when a ductile sacrificial layer structure was employed. The effect was demonstrated by Model 5 (hybrid) and contains about $10 \%$ more atoms than Model 2 (normal). Such an effect suggests the enhancement of plastic deformation and material removal rate for silicon when machined under the proposed hybrid condition. 


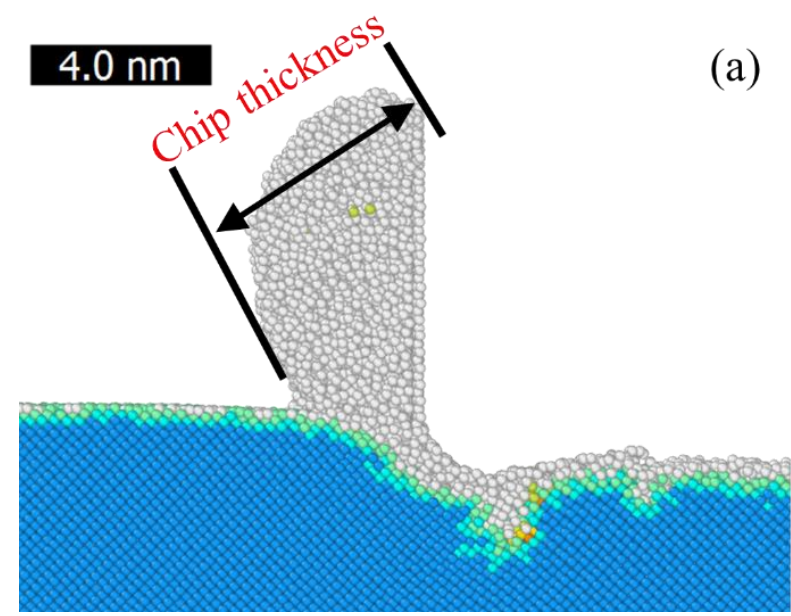

(a) $4.0 \mathrm{~nm}$

(b)

Figure 0-12: Cut chip thickness and subsurface deformation depth measurements in (a) Model 2 (Proposed hybrid condition) and (b) Model 5 (Normal condition).

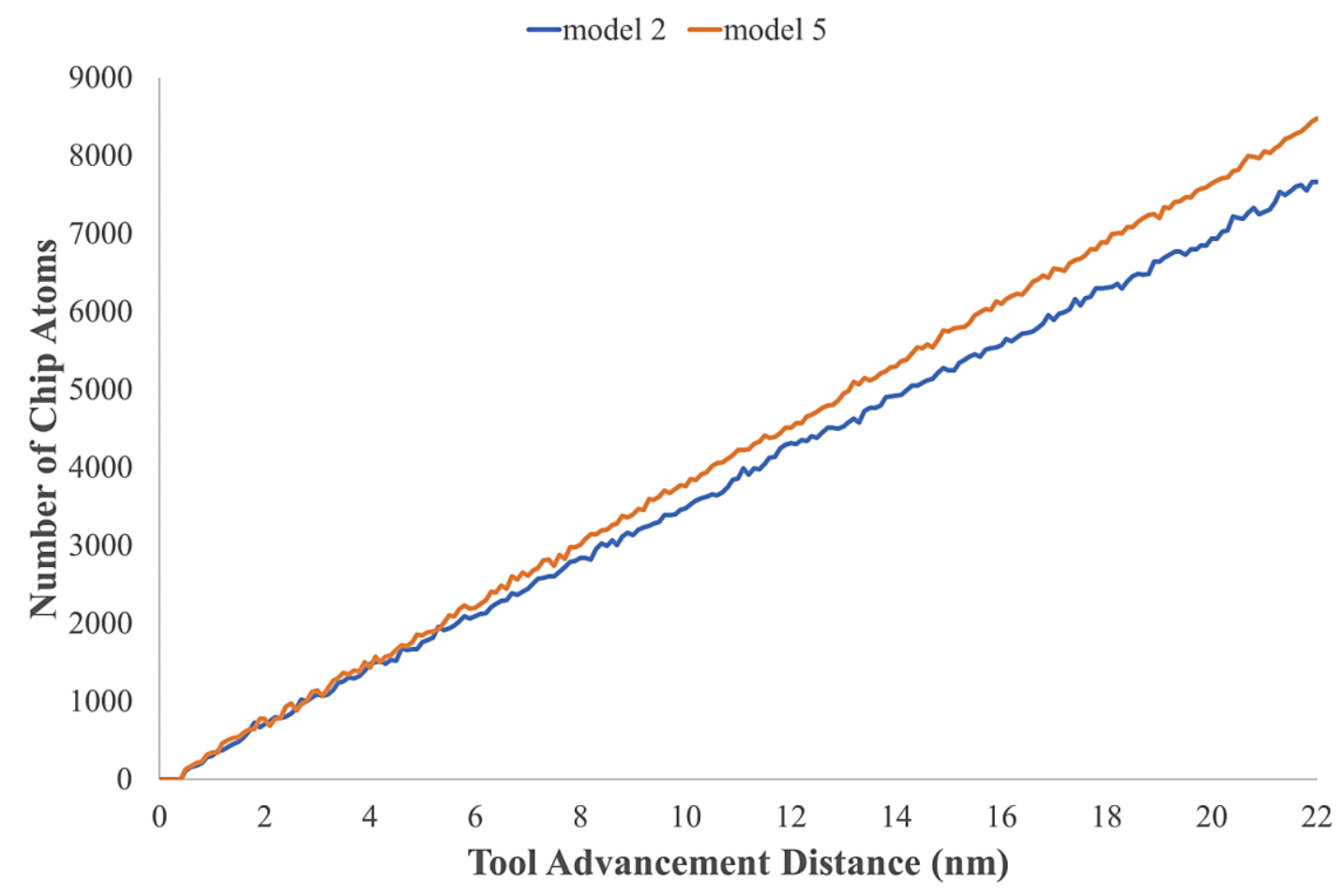

Figure 0-13: Number of atoms in the chip zone at tool travel distance of $25 \mathbf{n m}$ for Models 2 and 5. 


\subsection{Subsurface Deformation and Damage Depth}

Following the trail of assessments on the post-machining performance of silicon nanometric cutting, the depth of subsurface deformation and damage were quantitatively measured to determine the elastic deformation within the cutting zone and machined surfaces respectively. Figure 6-16 shows the chip morphologies and final machined surfaces of simulation Models 1, 2, 3 and 5 and 6 . Regions of the amorphous silicon structure underneath the tool cutting edge and along the machined surface denotes the subsurface deformation layer and the damage depth respectively. From the qualitative assessment, higher elastic deformation was observed in the cutting zone of Models 1 and 2 as shown in Figures 6-16(a) and 6-16(c) respectively, indicated by the larger subsurface deformation layer. Moreover, a thin protruding layer of amorphous silicon was observed along the final machined surfaces of Models 1 and 2 (Figures 6-16(b) and 6-16(d)). The effect represents a substantial "spring-back" due to large elastic deformation.

On the contrary, smaller subsurface deformation within the cutting zone and the domination of cubic crystalline structure along final machined surfaces as shown in Figures 6-16(e) to 6-16(j) were observed when silicon was machined under the proposed hybrid condition. Figure 6-17 also shows the measured values of both subsurface deformation and damage depths for all simulated models at a tool travel distance of $20 \mathrm{~nm}$ for more explicit depiction. Regardless of the machining feed direction and tool geometries, the subsurface deformation was reduced by up to $30 \%$, when silicon was machined under the hybrid condition. The effect was demonstrated by comparing the measured subsurface deformation depth for Models 2 (normal) and 3 (hybrid) at $1.786 \mathrm{~nm}$ and $1.271 \mathrm{~nm}$ respectively. Moreover, the subsurface damage was significantly reduced by $79 \%$ with the ductile sacrificial layer employment. The effect was demonstrated by comparing the measured subsurface deformation depth for Models 2 (normal) and 3 (hybrid) at $2.781 \mathrm{~nm}$ and $0.594 \mathrm{~nm}$ respectively.

Furthermore, the peculiarity of the anisotropic effect of silicon on the subsurface deformation and damage were minimised by the proposed hybrid technique. The effect was demonstrated by a reduction of up to $6 \%$ and $3 \%$ in the measured subsurface deformation and damage depths respectively when compared between Models 5 and 6 . The combinational effects obtained from the analysis of the subsurface deformation and damage indicate that higher elastic recovery can be achieved in silicon nanometric cutting by the proposed hybrid technique, thus resulting in higher shape accuracy upon machining. Observation of higher elastic recovery is particularly useful in ultra-precision machining and once again demonstrated the success capability of the proposed hybrid technique to improve silicon's machinability. 


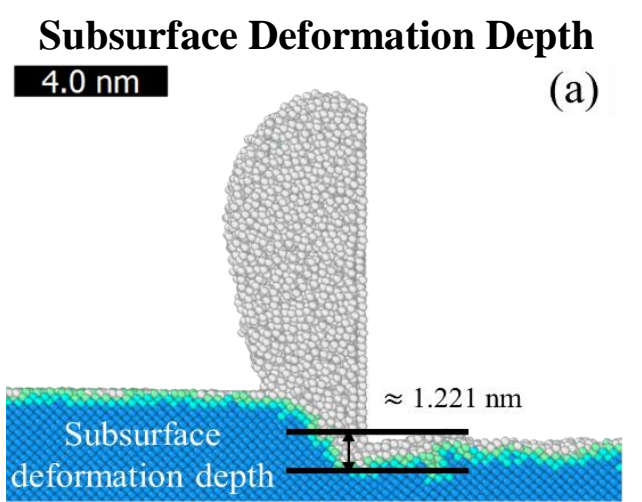

\section{$4.0 \mathrm{~nm}$}

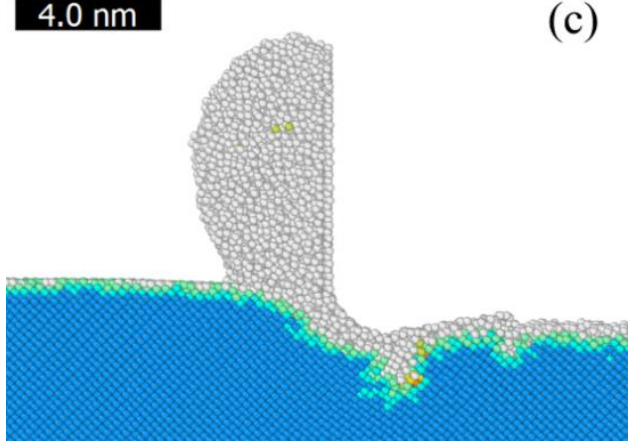

$4.0 \mathrm{~nm}$
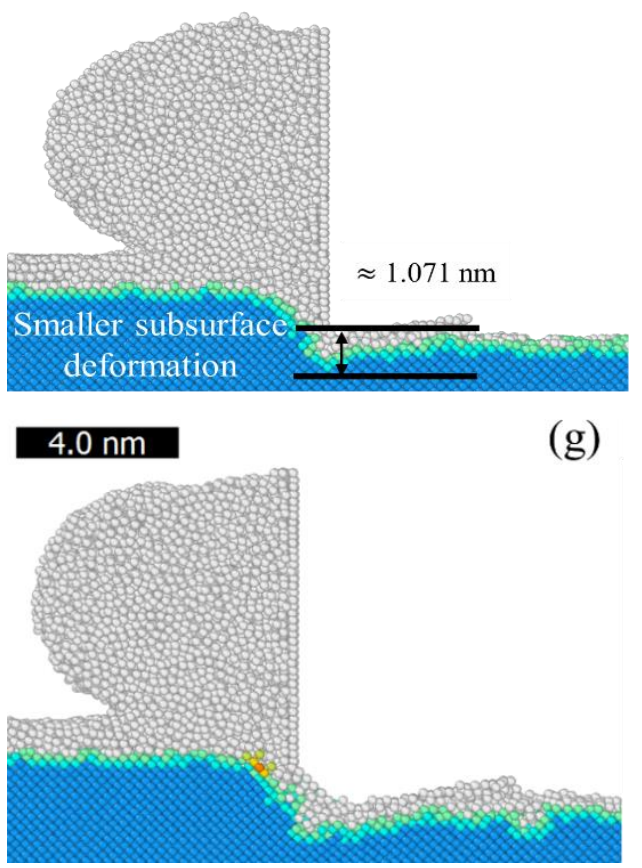

$4.0 \mathrm{~nm}$

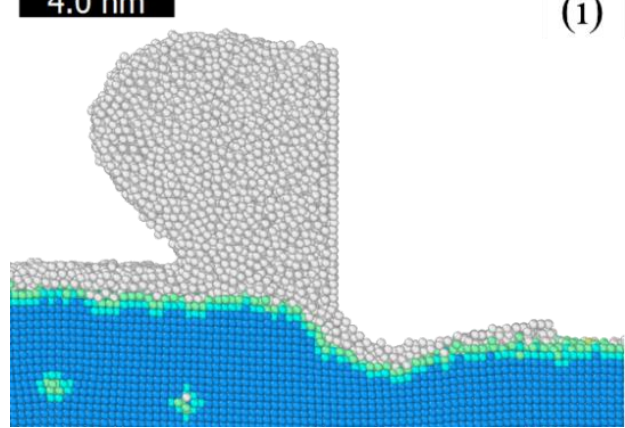

Subsurface Damage Depth

(b)
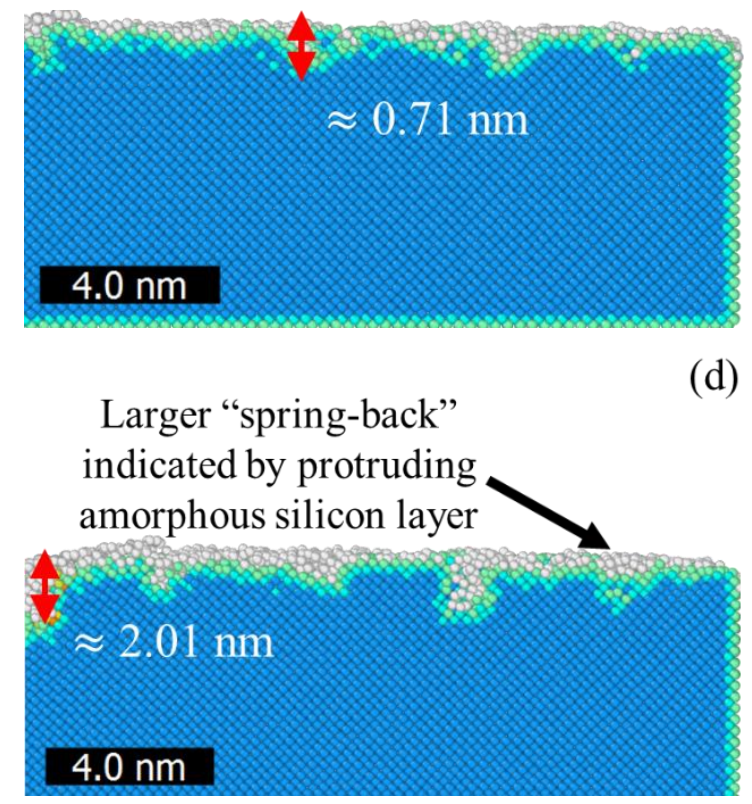

(f)

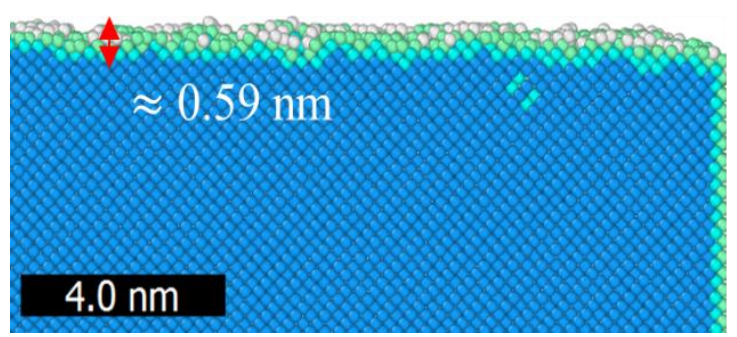

(h)

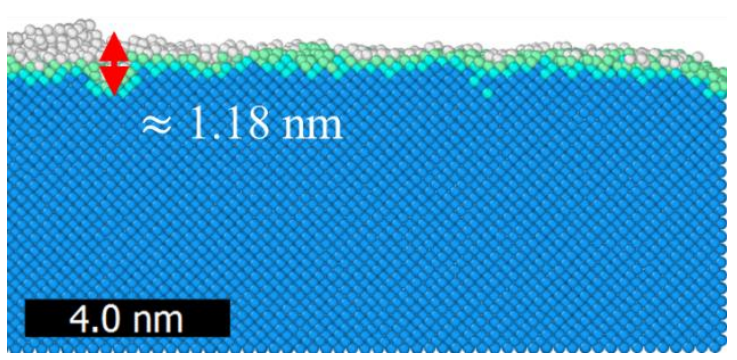

(j)

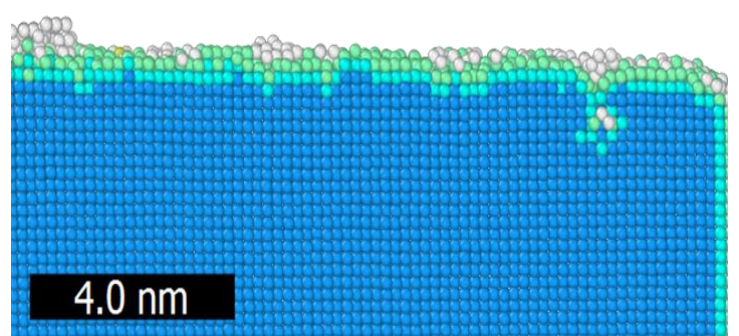

Figure 0-14: Chip morphologies and final machined surfaces of (a-b) Model 1, (c-d) Model 2, (e-f) Model 3, (g-h) Model 5 and (i-j) Model 6. 


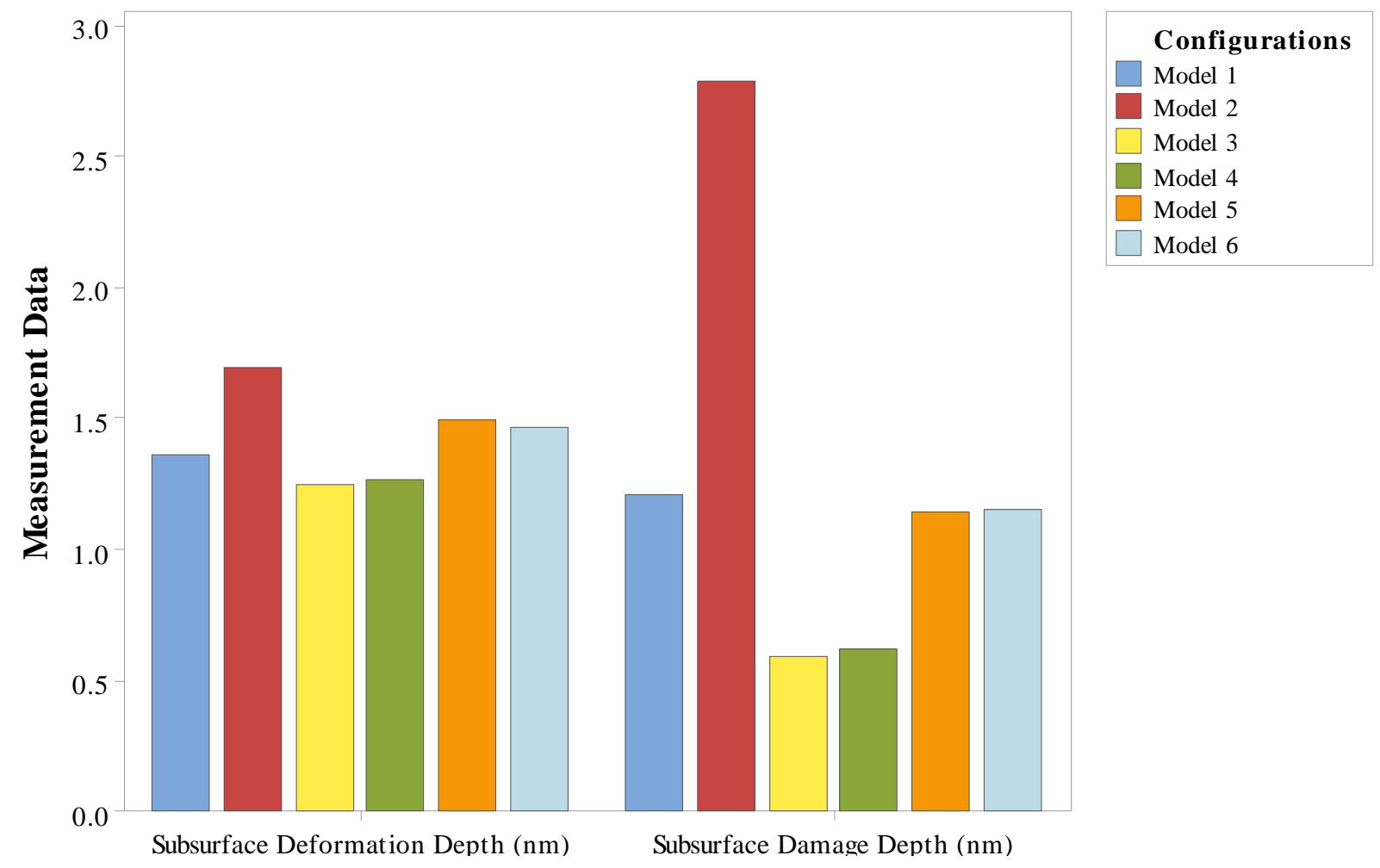

Figure 0-15: Summary of measured subsurface deformation and damage depth across all simulated models.

\subsection{Surface Morphology and Roughness Measurement}

From the wealth of literature in silicon nanometric cutting, general characterisation of postmachining performances usually involves subsurface structure and residual stress analysis [360]. However, there is also a growing interest in the measurement of surface roughness values on the final machined surfaces upon nanometric cutting in the MD simulation [361]. As such, this section aims to extract the nanometric morphologies of the final machined surfaces and compute its surface roughness value. As only a small region within the final machined surface can depict its morphology, the region of interest as shown in Figure 6-18 was used for the mesh construction to minimise the computational effort.

Figure 6-19 shows the selected surface morphologies of simulation Models 1, 2, 3 and 5. When monocrystalline silicon was machined under normal conditions, extreme surface irregularities and nano-pits can be observed through the surface morphologies of Models 1 and 2 as shown in Figures 6-19(a) and 6-19(b) respectively. The intensity of the surface irregularity and defects were also higher in the case of Model 2, where silicon was machined by a rounded cutting edge tool.

As discussed, the use of a tool with larger cutting edge radius can increase the cutting resistance between tool cutting edge and workpiece, and eventually affects the chip formation and surface 
generation processes. On the contrary, the employment of a ductile sacrificial layer structure under the proposed hybrid technique framework reduces the surface irregularities and defects to be minimised, thus resulting in the improvement of final machining quality.

The effect was depicted by the relatively smoothed surface contours that were demonstrated by the surface morphologies of Models 3 and 5 as shown in Figures 6-19(c) and 6-19(d). Quantitative measurements of the surface roughness values for all simulated models are also shown in Figure 6-20. The surface roughness value in this study was quantitatively evaluated by the root mean square roughness value, $R_{a}$, computed using Equation 6-2 [362].

$$
R_{a}=\frac{1}{N} \sqrt{\sum_{i=1}^{n}\left(Y_{i}-\bar{Y}\right)^{2}}
$$

Where

- $Y_{i}$ represents the height of the exposed atoms above the theoretical surface threshold plane along the Y-axis.

- $\bar{Y}$ is the mean height of all the exposed atoms.

- $\quad N$ is the total number of exposed atoms.

The theoretical surface threshold plane as described in Equation 6-2 is defined by the theoretical height of the final machined surface. The theoretical height value of the final machined surface can be obtained by subtracting the height of the workpiece with the cutting depth before the cutting process. A precise depiction of the described theoretical surface threshold plane and height are shown in Figure 6-21.

According to the computation, the use of a ductile sacrificial layer structure enables a significant decrease in the surface roughness value of up to $60 \%$ can be observed when silicon was machined by an atomically sharp tool along the [100] directions under the proposed hybrid condition. The effect was demonstrated by the $R_{s}$ of Model 3 (hybrid) at $0.098 \mathrm{~nm}$ when compared to the $R_{a}$ value of Model 1 (normal) at $0.236 \mathrm{~nm}$.

Moreover, the measurement also illustrates an expected behaviour whereby the machining of silicon by a tool with a rounded cutting edge yields poorer machining quality, regardless of other machining conditions. However, unlike the subsurface deformation and damage depths measurements, the influence of the machining feed directions shows a distinctive anisotropic behaviour in the surface roughness values. An increment of about $12 \%$ in the surface roughness values can be observed when machining was conducted along the [110] direction with either an 
atomically sharp or rounded cutting tool under the proposed hybrid machining conditions. The effect was demonstrated by comparing between the computed $R_{s}$ values of Models 3 and 4 at $0.098 \mathrm{~nm}$ and $0.111 \mathrm{~nm}$ respectively, and between the computed $R_{s}$ values of Models 5 and 6 at $0.145 \mathrm{~nm}$ and $0.164 \mathrm{~nm}$ respectively.

The effect was believed to be attributed by the consideration of more datasets extracted in a three-dimensional environment for the surface roughness value computation, as compared to the respective subsurface measurements performed on a two-dimensional cross-section view of the machined workpiece. Despite so, the influence of the machining feed direction on silicon nanometric cutting by the hybrid technique was considered small and negotiable. Furthermore, it is worth noting that the computed surface roughness values can only represent an indicative assessment rather than the actual attainable experimental values. This is due to the dimensional limitation of MD simulation.

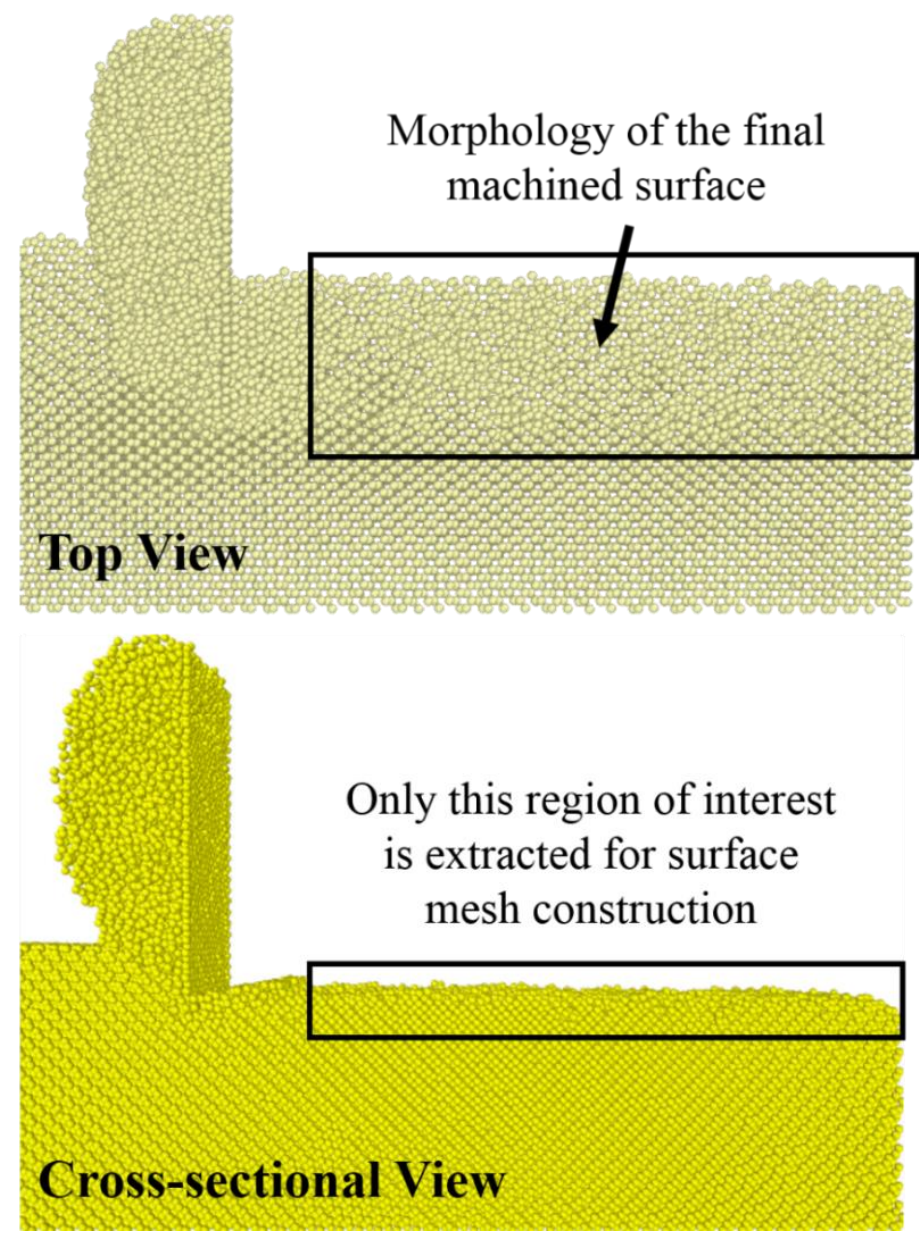

Figure 0-16: Illustration on the region of interest along the final machined surface extracted for surface mesh construction and surface morphology visualisation. 


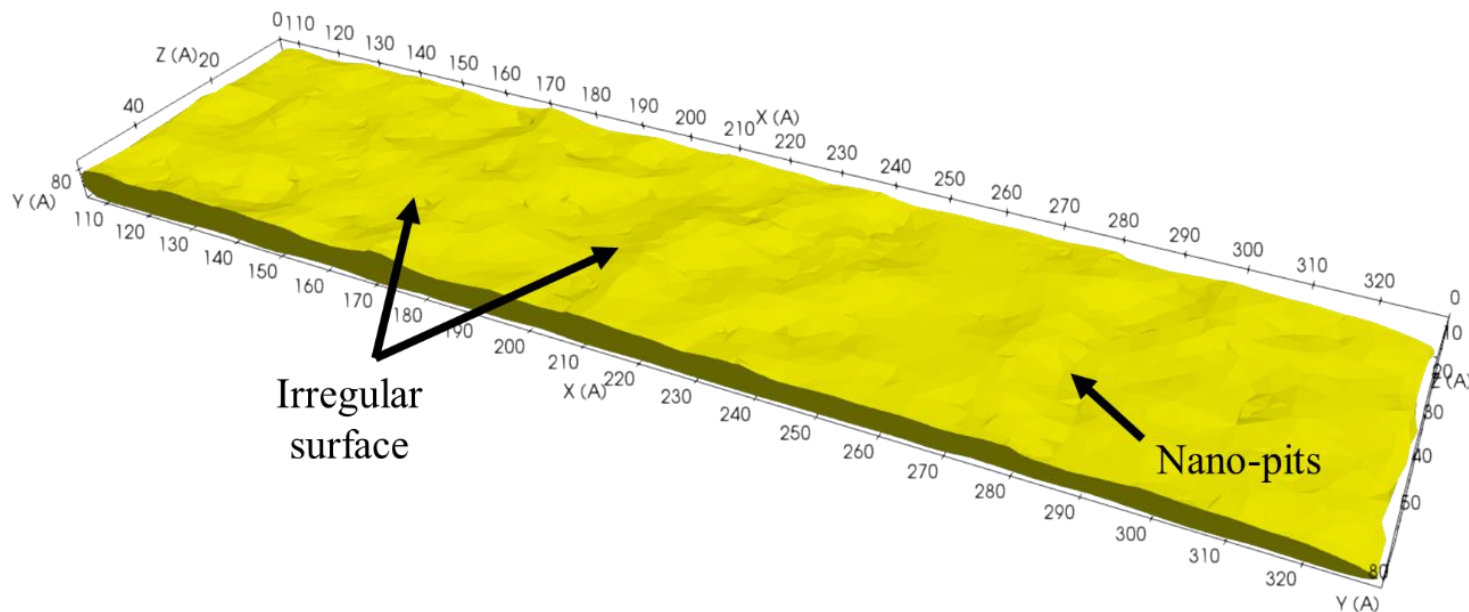

(a)

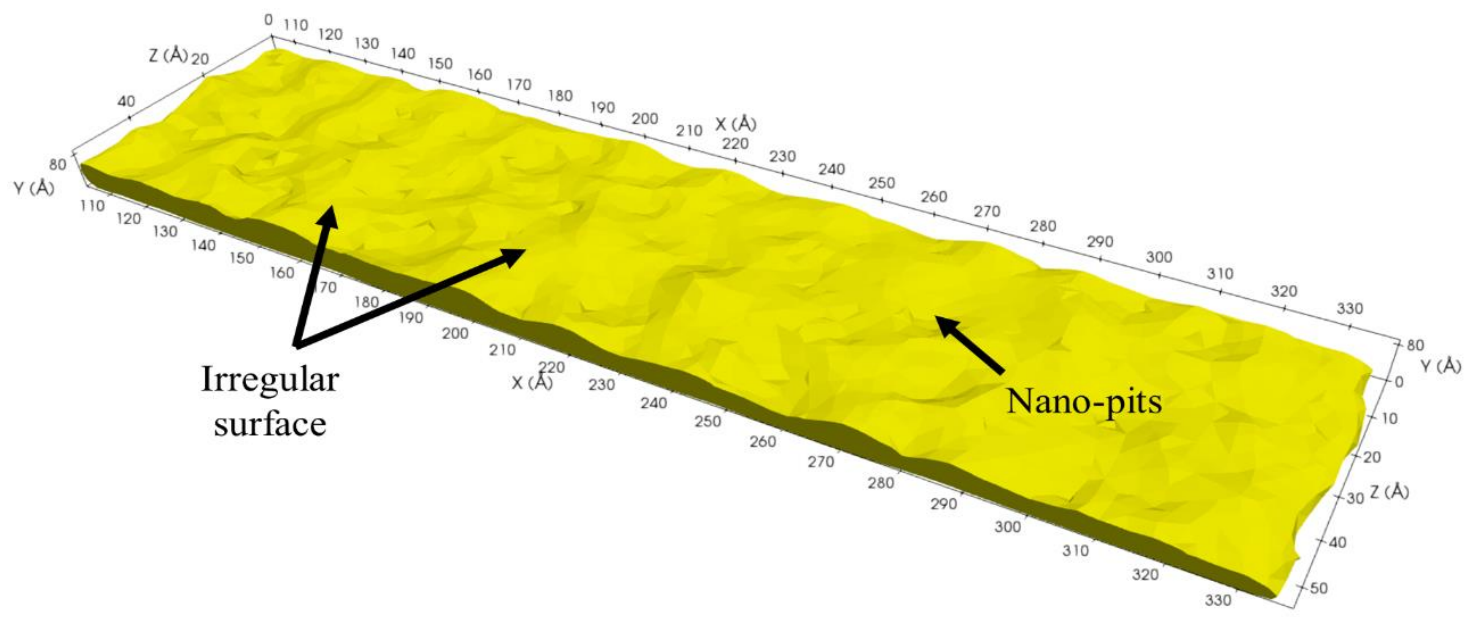

(b)

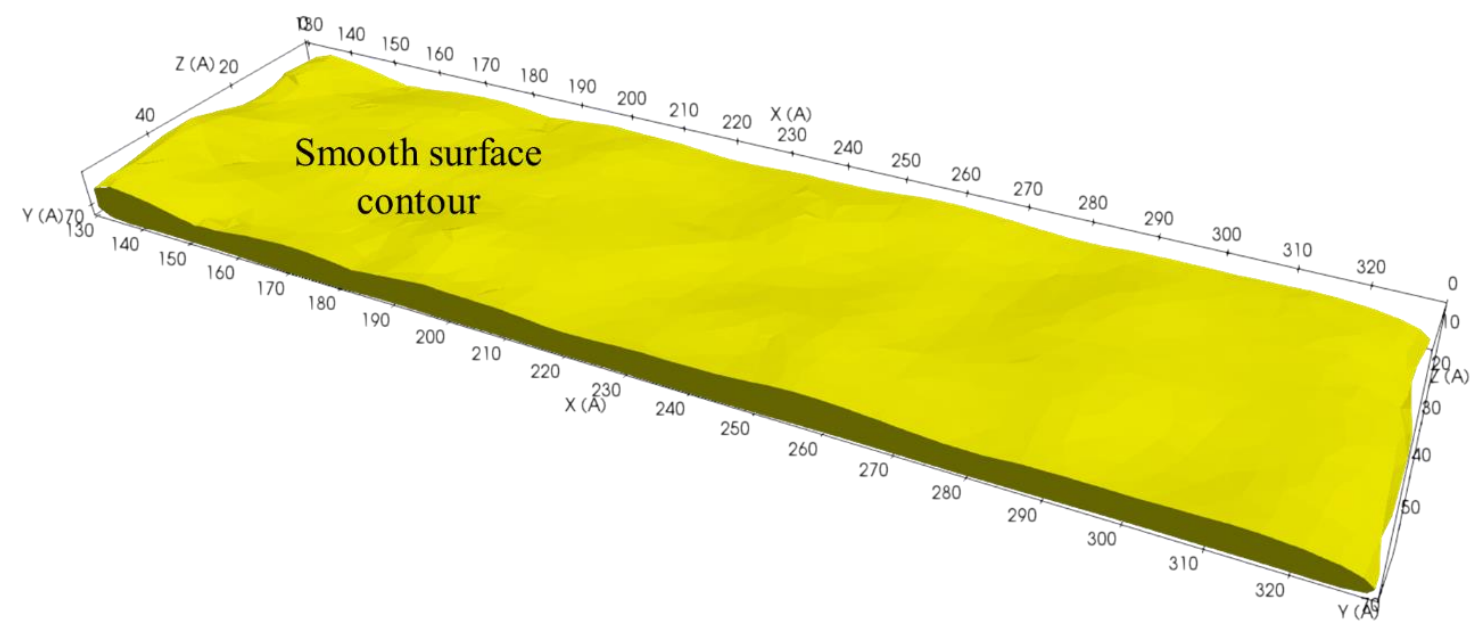

(c) 


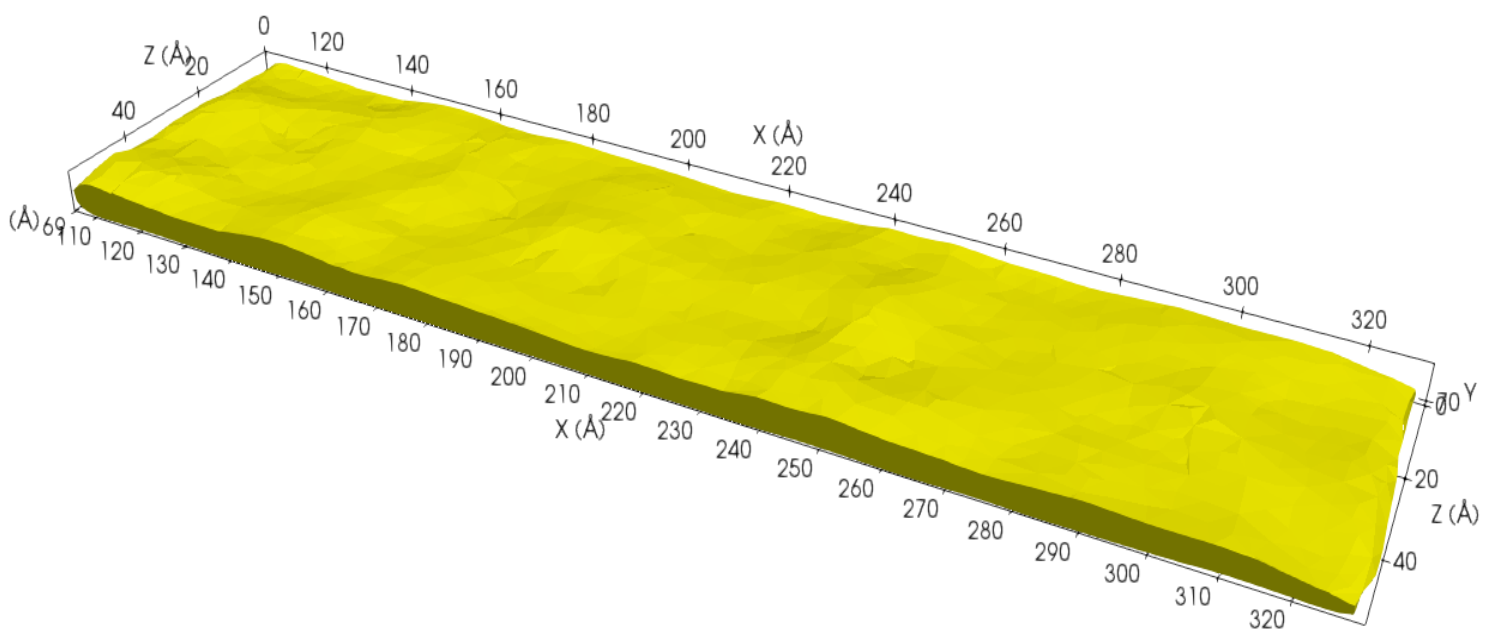

(d)

Figure 0-17: Constructed surface morphologies of the final machined monocrystalline silicon surfaces in (a) Model 1, (b) Model 2, (c) Model 3 and (d) Model 5.

\subsection{5}

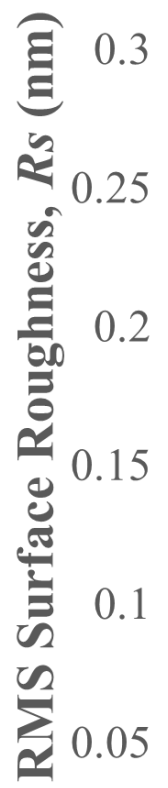

0

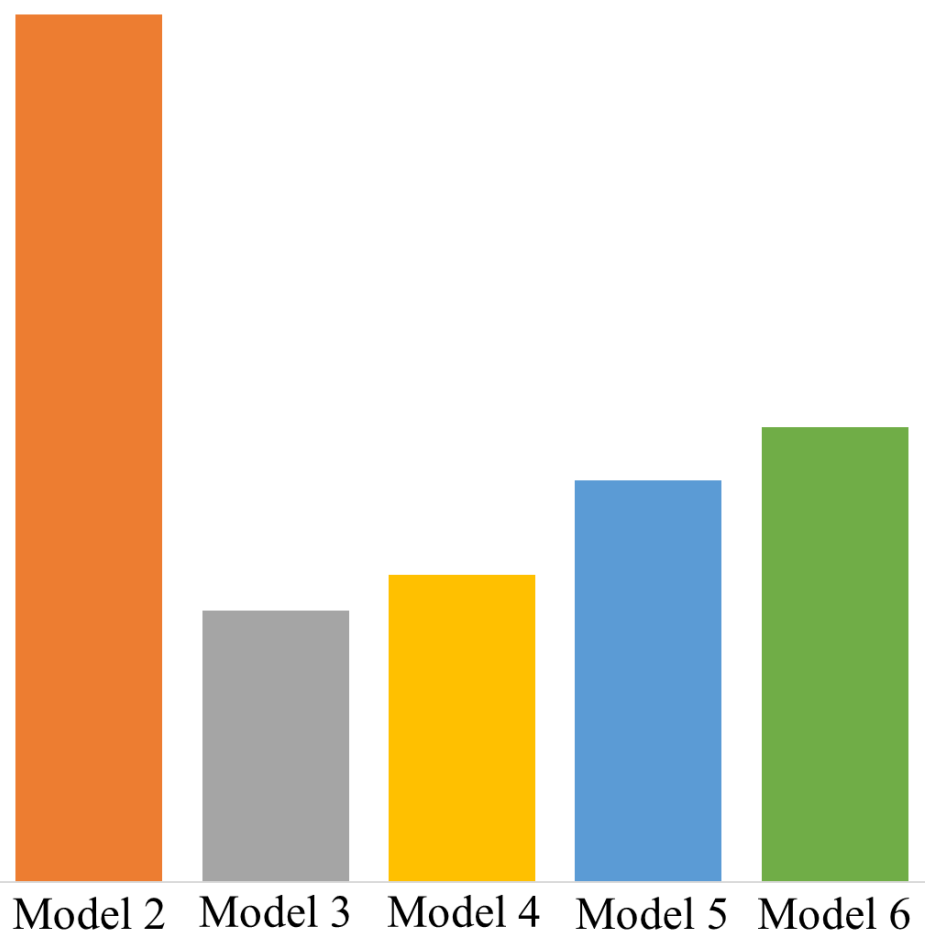

Figure 0-18: Summary of root mean square surface roughness value computed across all simulation models. 


\section{Tool}

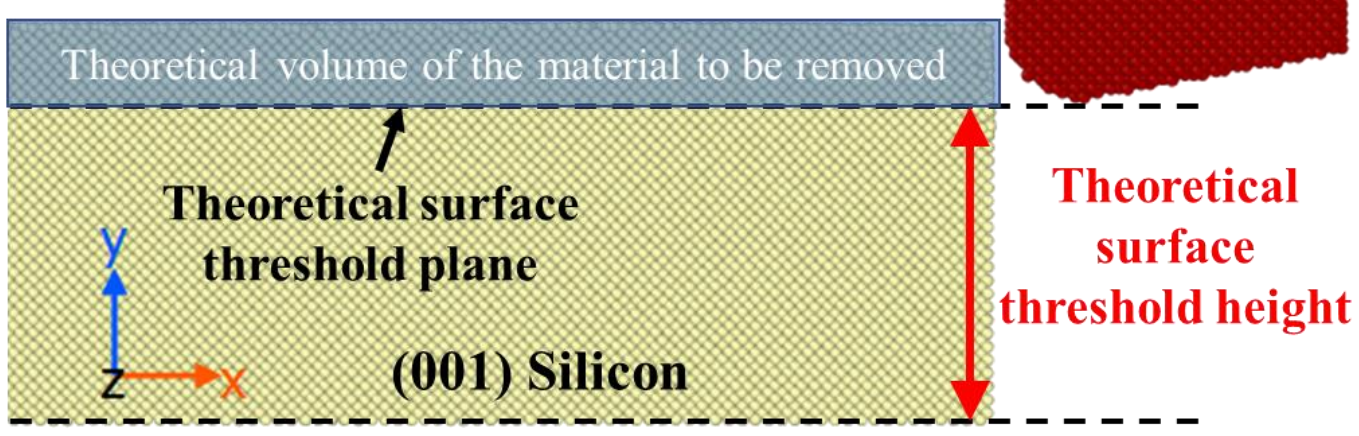

Figure 0-19: Clearer depiction of the theoretical surface threshold plane and height required in the computation of root mean square surface roughness value.

\section{Conclusion}

In this paper, a novel hybrid manufacturing technique combining ultra-precision machining with a deposition process to create a ductile sacrificial layer for brittle silicon fabrication has been proposed. The hybrid technique aims to be useful in reducing edge chipping during the fabrication of brittle materials such as the silicon-based implant while preserving the surface and structural properties on the unmachined regions of the finished product. Based on the proposal, a study to investigate the cutting mechanism of monocrystalline silicon under the proposed hybrid machining conditions has been presented.

Reported results from this study had demonstrated an early success of employing the proposed novel hybrid manufacturing technique. The success has been shown in various performances analysis such as machining stress, forces, cutting chips, subsurface measurements and surface roughness values computation. Based on these analyses, the main conclusions are summarised as follows:

1. It was evident that the influence of the sacrificial layer causes its ductile characteristics to dominate over the brittleness of silicon in the amorphous-crystalline composition region, leading to plasticity enhancement of the silicon substrate. The effect produces a significant decrease in the hydrostatic pressure, maximum shear stress, and atomic strain within the cutting zone. This was due to the better deformability of the ductile sacrificial layer structure and thermal softening of the workpiece from the massive heat generated by the high interfacial stress between the sacrificial layer structure and the silicon substrate. The 
outcome of such effect leads to the generation of minimal subsurface deformation and damage depth and signifies the minimisation of silicon edge chipping by the hybrid technique.

2. When silicon nanometric cutting was performed under the proposed hybrid machining conditions, a relatively stable cutting force was also observed. Such observation was due to the reduction of cutting resistance attributed to plasticity enhancement, which in turn correlates with the lower concentration of tensile stress in the silicon workpiece. Moreover, reduction of tensile stress also signifies the reduced risk of edge chipping development and propagation on post-machined surfaces.

3. Subsequently, promising results demonstrated from the chips, subsurface measurements and surface roughness values computation indicate the successful employment of the proposed hybrid technique. Smaller elastic deformation, higher material removal rate and improved surface finishing of $<0.1 \mathrm{~nm}$ were achieved. Moreover, although better machining performances were achieved along the [100] direction, the anisotropic influence of silicon crystal structure was significantly minimised by the proposed hybrid conditions. Such an effect will be useful in the machine path planning for the shaping of advanced geometries. 
References

5-10 put some ductile mode cutting silicon paper

11-12 put some HPPt references

13-17 references for vibration, thermal (laser), chemical and ion implantation

18-20 chemical, laser assisted and vibration assisted challenges 